\title{
Bernd Lindner
}

\section{„Bau auf, Freie Deutsche Jugend“ - und was dann? Kriterien für ein Modell der Jugendgenerationen der DDR}

"Generationen werden heute", so das Ergebnis einer Tagung über die neue Warenwelt und deren Folgen für das individuelle Bewußtsein in der Gegenwartsgesellschaft, „nicht mehr nach historischen Daten, sondern nach einer Automarke benannt. Leute definieren sich selbst weniger über Konfessionen, politische Lager, soziale Klasse oder ethnische Zugehörigkeit als über ihre persönliche Markenkultur. "I Für eine Klassifizierung der Jugendgenerationen der DDR kommt diese Erkenntnis der "Generation Golf“ 2 nicht nur zu spät, sie wäre zudem auch kaum glaubhaft zu vermitteln. In einer Mangelgesellschaft wie der DDR - und das war sie durchgängig bis zu ihrem Ende ${ }^{3}$ - war eine produktinspirierte Generationenprägung undenkbar, zumal sich die Warenwünsche vieler Jugendlicher im Osten Deutschlands sehnsuchtsvoll (und oft unstillbar) an Markenprodukten des Westens und nicht an den Waren der eigenen Konsumgüterproduktion ausrichteten. Als Ulrich Plenzdorf Anfang der 1970er Jahre den Helden seines Erfolgsstückes "Die neuen Leiden des jungen W.“, Edgar Wibeau, den Satz: „Ich meine, Jeans sind eine Einstellung und keine Hosen "4 von den Bühnen der DDR verkünden ließ, dachte mit allergrößter Wahrscheinlichkeit keiner der jungen Zuschauer im Saal an "Bison-" oder "Wisent-Jeans" aus der volkseigenen Produktion.

Statt der (neu)modischen Definition über Markenprodukte bietet sich für die Jugendgenerationen der DDR eine Kennzeichnung ihrer unterschiedlichen Generationsgestalten durch jene zentralen Aktivitätsformen an, mit denen sie in ihrer

1 Zitiert nach Süddeutsche Zeitung vom 3. Mai 2000: Eva Marz, Glück kaufen. Die Wahrheit der Warenwelt (Bericht über eine Tagung an der Evangelischen Akademie Tutzing).

2 Florian Illies, Generation Golf. Eine Inspektion (Berlin 2000).

${ }^{3}$ Für die ostdeutsche Ethnologin Ina Merkel lagen die Grundprobleme in der Versorgung der Bevölkerung in der nunzulängliche(n) Befriedigung der Bedürfnisse aufgrund von Mängeln des Angebots hinsichtlich Menge, Sortimentsstruktur, Neuheiten, Qualität und Verpakkung. Diese Mängel äußerten sich nicht nur als Sortimentslücken und Engpässe, sondern auch als ihr Gegenteil, Überangebot und Ladenhüter". In: Utopie und Bedürfnis. Die Geschichte der Konsumkultur in der DDR (Köln, Weimar, Wien 1999) 89.

4 Ulrich Plenzdorf, Die neuen Leiden des jungen W. (Rostock 1973) 20. 
Jugendzeit die Gesellschaft und sich selbst am nachhaltigsten geprägt haben. Demnach zeichnen sich für den Zeitraum von 1945 bis 19895 - im Sinne des Mannheim'schen Generationenkonzeptes ${ }^{6}$ - im Osten Deutschlands meines Erachtens drei prägende Generationseinheiten ab, die in ihrer historischen Abfolge gekennzeichnet waren durch

- ihre aktive Beteiligung am Aufbau des ersten "sozialistischen Staates der Arbeiter und Bauern" auf deutschem Boden' in der Jugendgeneration der unmittelbaren Nachkriegszeit und in der Gründungsphase der DDR;

- ihr anhaltendes Mitmachen bei der weiteren „Gestaltung der entwickelten sozialistischen Gesellschaft ${ }^{4}$, trotz vieler Warnzeichen und punktuell wachsender Zweifel an der selbstangemaßten Legitimität der SED zur Alleinherrschaft in der zweiten ost deutschen Jugendgeneration; sowie

- ihre zuerst innere, in der Endphase der DDR dann auch massenhaft physisch vollzogene Distanzierung der dritten ostdeutschen Jugendgeneration vom "real existierenden Sozialismus ${ }^{49}$ statischen Zuschnitts der gerontokratischen SEDFührung.

Im saloppen Idiom heutiger Jugendsprache ließe sich die Abfolge der Jugendgenerationen im Osten Deutschlands etwas lax, aber dennoch treffend auf die Schlagworte - Bau auf, Mach mit, Hau ab! - bringen. Wissenschaftlich definiert, wäre sie als Übergang von der Aufbaugeneration zu einer Integrierten Generation hin zu einer mehrheitlich Distanzierten Generation zu beschreiben.

Diese dreigliedrige Generationenfolge erfaßt selbstverständlich nur den Mainstream der Jugendentwicklung in den unterschiedlichen historischen Phasen der SBZ/DDR, deren jeweiliger politischer, kultureller und sozialer Charakter den zentralen Generationseinheiten des Landes seinen Stempel aufgedrückt hat, wie auch sie ihrerseits diese unterschiedlichen Phasen aktiv mit geprägt haben. Ihnen zur Seite bzw. partiell auch konträr gegenüber standen in den selben Generationszusammenhängen jedoch stets weitere Generationseinheiten ${ }^{10}$, die es ebenfalls zu benennen gilt, will man ein umfassendes Bild von der Entwicklung der Jugend in der DDR geben; auch wenn nicht jede von ihnen prägenden (und damit auch namensgebendenen) Charakter für "ihre" Generation zu erlangen vermochte. Dennoch sind sie als Widersacher gegen den Mainstream für dessen „Prägnanzbil-

${ }^{5}$ Die vier Jahre Sowjetische Besatzungszone (SBZ) von 1945 bis 1949 gehören unmittelbar zur Vorgeschichte der DDR und können daher bei der Konstruktion eines Modells der Jugendgenerationen des Landes nicht ausgeklammert werden.

6 Vgl. Karl Mannheim, Das Problem der Generationen, in: ders., Wissenssoziologie. Auswahl aus dem Werk, eingeleitet und hrsg. v. Kurt $H$. Wolf (Darmstadt 1964) 509-565; im folgenden zitiert: Mannheim, Generationen.

7 Artikel 1 der Verfassung der DDR von 1949, Neufassung 1968.

${ }^{8}$ Erich Honecker, Bericht des Zentralkomitees der Sozialistischen Einheitspartei Deutschlands an den VIII. Parteitag der SED (Berlin 1971) 9.

${ }_{9}$ Erich Honecker, Bericht des Zentralkomitees der Sozialistischen Einheitspartei Deutschlands an den XI. Parteitag der SED (Berlin 1986) 7.

10 Mannheim, Generationen 541-544. 
dung" (Niethammer) unabdingbar"1. Gleichzeitig ist zu betonen, daß es nicht immer die nominell stärkste Generationseinheit sein muß, von der diese prägende Kraft ausgeht. Im Gegenteil: Es sind - wie Heinz Bude zu Recht betont - mitunter nur wenige, „die dem Gefühl und der Gesinnung einer Generation Ausdruck verleihen, aber die Generationen unterscheiden sich danach, in welchem Umfang das dominierende Deurungsmuster die Jahrgangsgruppe insgesamt trifft ${ }^{*}{ }^{12}$.

Die Abfolge und der Wandel der DDR-Jugendgenerationen sollen im vorliegenden Beitrag für den Zeitraum von 1945 bis 1989 überblicksartig dargestellt werden. Im Vordergrund steht dabei das ,intragenerationelle, synchrone Verhältnis der Jüngeren untereinander", während die „intergenerationelle, diachrone Konfliktlage ${ }^{\text {"13 }}$ zwischen jüngeren und älteren Kohorten in der SBZ/DDR (aus Platzgründen) hier nur bedingt ausgeführt werden kann, ohne damit die Bedeutung von Reverenzgenerationen für die Ausprägung neuer Generationsgestalten unterbewerten zu wollen.

\section{Modelle und Motivationen}

Wer sich heute mit den generationellen Prägungen der Jugendgenerationen der DDR auseinandersetzen will, trifft auf eine beachtliche Zahl analoger Versuche. Das war nicht immer so. In der DDR wurde das Generationskonzept innerhalb der Gesellschaftswissenschaften nahezu vollständig ausgegrenzt. W'eder in der (Sozial-)Psychologie noch in der Soziologie oder der Jugendforschung konnte es zum Tragen kommen. Zu groß war die Furcht der Herrschenden, daß mit diesem Thema zugleich auch die von ihnen verdrängten Konflikte zwischen den Generationen zur Sprache kommen würden ${ }^{14}$. Lediglich in der Literaturwissenschaft gelang es (wenn auch spät), ansatzweise dem Befund Rechnung zu tragen, „da13 Generationsunterschiede eine gesetzmäßige Lebenstatsache sind ${ }^{415}$ - eine

11 Mannhcim verweist ebenfalls darauf, daß sich „im Rahmen desselben Generationszusammenhanges ... mehrere, polar sich bekämpfende Generationseinheiten bilden “, die gerade dadurch einen "Zusammenhang“ bilden, "daß sie aufeinander, wenn auch kämpfend, abgestimmt sind". (Mannbeim, Generationen 547).

12 Heinz Bude, Das Altern einer Generation. Die Jahrgänge 1938-1948 (Frankfurt a.M. $1997) 47$.

13 Thomas Köbler, Jugendgenerationen im Vergleich: Konjunkturen des (Non-)Konformismus, in: APuZ B5 (1. Februar 2002) 7.

${ }^{14}$ So wird im "Worterbuch zur Sozialistischen Jugendpolitik“, unter dem Stichwort Generation, die $"$ von antikommunistischen Ideologen vertretene These eines allgemeinen Generationenkonflikts ... als unwissenschaftlich" abgelehnt. Der "Versuch, einen ,Generationskoflikt' innerhalb der sozialistischen Gesellschaft zu konstruieren, ist von dem Streben der imperialistischen Politik getragen, die Jugend der sozialistischen Länder in Widerspruch zur älteren Generation, besonders aber zur Partei der Arbeiterklasse und ihrer bewährten Führung, zu bringen“", (Berlin 1975) 87; im folgenden zitiert: Wörterbuch Jugendpolitik.

15 Hans Richter (Hrsg.), in: Generationen - Temperamente - Schreibweisen. DDR-Literatur in neuer Sicht (Halle 1986) 7. Vgl. auch ders., Zum Thema Generation(en), in: Temperamente. Blätter für junge Literatur 1 (1989) 2-15. 
Erkenntnis, die bis dahin einzig in den Künsten kontinuierlich reflektiert worden ist ${ }^{16}$.

Seit der Gesellschaftswende von 1989/90 wird das Thema aus vielfältigen geistesund sozialwissenschaftlichen Perspektiven bearbeitet. Zeitgeschichtler bemühen das Generationenkonzept ebenso wie Soziologen, Philosophen, Erziehungswissenschaftler oder Kunsthistoriker. Eine Begründung für diese neuerliche Konjunktur findet sich bereits in der Grundlegung des Ansatzes bei Mannheim, sah er doch in der generationsgeleiteten Gesellschaftsanalyse einen „unerläßlichen Führer" bei der Erkenntnis des Aufbaus sozialer und geistiger Bewegungen: „Seine praktische Bedeutung wird unmittelbar ersichtlich, sobald es sich um das genauere Verständnis der beschleunigten Umwälzungserscheinungen der unmittelbaren Gegenwart handelt. ${ }^{~}{ }^{17}$ Und mit solchen "beschleunigten Umwälzungserscheinungen ${ }^{*}$ hatten wir es in den letzten zwölf Jahren im Osten Deutschlands zweifelsohne zu tun. Sie in ihrem Charakter zu erfassen und zu deuten, ist auch mein Anliegen. Es geht mir bei der Anwendung des Generationskonzepts auf die Jugendentwicklung in der DDR also nicht allein um rückwärts gewandte Erklärungsversuche eines überwundenen politischen Systems und seines Unterganges, sondern auch um eine Deutung gegenwärtiger Befindlichkeiten aus ihrer historischen und soziologischen Genese heraus. Zugleich speist sich mein Engagement für diesen Analyseansatz aus meinem eigenen wissenschaftlichen Werdegang als Jugendforscher und Zeithistoriker: Von 1978 bis 1992 war ich als Kultursoziologe in der Jugendforschung tätig: zuerst - bis zu dessen verordneter Auflösung Ende 1990 - am Zentralinstitut für Jugendforschung (ZIJ) in Leipzig; weitere zwei Jahre dann an der Außenstelle des Deutschen Jugendinstituts München (DJI) am selben Ort. Zugleich beschäftigte ich mich seit 1990 als Wissenschaftler wie beteiligter Zeitzeuge mit der historischen Aufarbeitung der DDR-Gesellschaft und ihres jähen Endes ${ }^{18}$, ohne dabei jedoch den "soziologischen Blick" vollkommen abzustreifen ${ }^{19}$.

Seit 1994 bin ich hauptberuflich am Aufbau des Zeitgeschichtlichen Forum Leipzig beim Haus der Geschichte der Bundesrepublik Deutschland - also einem zeitgeschichtlichen Museum, das der Geschichte der DDR und der Opposition gegen sie, wie auch der Geschichte des geteilten Deutschlands gewidmet ist ${ }^{20}-$ be-

16 Vgl. Bernd Lindner, Väter - Söhne - Mütter - Töchter. Der Generationskonflikt bei Christoph Hein, Volker Braun, Uwe Saeger und Heiner Müller, in: DDR-Literatur ' 89 im Gespräch, hrsg. v. Siegfried Röbnisch (Berlin, Weimar 1999) 123-135.

17 Mannheim, Generationen 522.

18 Vgl. dazu u. a.: Bernd Lindner (Hrsg.), Zum Herbst '89. Demokratische Bewegung in der DDR (Leipzig 1994); ders., Die demokratische Revolution in der DDR 1989/90 (Bonn 1998); im folgenden zitiert: Lindner, Demokratische Revolution.

19 Vgl. dazu u.a.: Demonteure. Biographien des Leipziger Herbst, hrsg. v. Bernd Lindner, Ralph Grüneberger (Bielefeld 1992); Bernd Lindner, Verstellter, offener Blick. Eine Rezeptionsgeschichte bildender Kunst im Osten Deutschlands 1945-1995 (Köln, Weimar, Wien 1998).

20 Vgl. dazu: Stiftung Haus der Geschichte der Bundesrepublik Deutschland/Zeitgeschichtliches Forum Leipzig (Hrsg.), Einsichten. Diktatur und Widerstand in der DDR (Leipzig 2001) 7 ff.; im folgenden zitiert: ZFL, Einsichten. 
teiligt. Auf all diesen Ebenen waren und sind Jugendliche und ihre generationellen Prägungen Gegenstand meiner wissenschaftlichen Arbeit ${ }^{21}$. So fließen in meiner Berufsbiographie zwei Wissenschaftsstränge ineinander, die in den bisher vorliegenden Analysen der DDR-Gesellschaft meist weitgehend getrennt voneinander behandelt worden sind. Während Soziologen das überraschende Ende der DDR vor allem aus den Verlaufsreihen empirischer Daten zu analysieren suchen, sind Historiker wie auch Politikwissenschaftler stärker auf die von ihr hinterlassenen Archivalien und Zeitzeugnisse fixiert. Eine Zusammenführung dieser unterschiedlichen Quellen ist bisher kaum versucht worden.

Zugleich ist bei den vorliegenden Analysen der DDR-Gesellschaft immer wieder eine gewisse Parzellierung der Betrachtungsweisen zu verzeichnen. Geht es Politikwissenschaftlern und Zeithistorikern verstärkt um die Herrschaftsgeschichte des SED-Staates ${ }^{22}$, stellen Soziologen und Kulturwissenschaftler prononciert die Mentalitätsgeschichte der Ostdeutschen ${ }^{23}$ in den Mittelpunkt ihrer Betrachtungen. Oft gelingt es im Ergebnis aber nicht, das Verhältnis von Alltag und Herrschaft in dem nötigen Maße miteinander zu verquicken²4. Dadurch erscheinen viele der Deutungsversuche entweder eigenartig menschenleer oder verlieren durch eine überzogene Fokussierung auf die handelnden Individuen den notwendigen Kontakt zum politischen Herrschaftsrahmen. Die Geschichte der $D D R$ mit all ihren Brüchen ist jedoch weder durch die eine noch durch die andere Betrachtungsweise allein hinlänglich zu fassen. Meines Erachtens muß sie als ein Prozeß der zunehmenden Gewinnung von Handlungsspielräumen durch die Individuen gegenüber einem zentralistisch verfaßten Staatswesen gesehen werden, welches auf der einen Seite ohne die Einsatzbereitschaft und Kreativität seiner Bürger nicht existieren konnte, auf der anderen Seite aber - aus einer totalitären Staaten eigenen Bevormundungshaltung heraus - die dafür nötigen Freiräume nicht gewähren wollte. Die DDR konnte als "Land der kleinen Leute“ - wie sie

21 Zuletzt in der von mir kurartierten Ausstellung „Foto-Anschlag. Vier Generationen ostdeutscher Fotografen“, Zeitgeschichtliches Forum Leipzig (12.7.-10.10. 2001) und Haus der Geschichte Bonn (15. 5.-28. 8. 2002). Das gleichnamige Begleitbuch zur Ausstellung hrsg. v. der Stiftung Haus der Geschichte der Bundesrepublik Deutschland - ist 2001 in Leipzig erschienen.

22 S. ctwa die Materialien der Enquete-Kommission zur Aufarbeitung von Geschichte und Folgen der SED-Diktatur in Deutschland (Baden-Baden 1995 und 1999; insgesamt 17 Bände) oder Klaus Schröder in seiner Monographie: Der SED-Staat. Geschichte und Strukturen der DDR (München 1999).

23 S. Wolfgang Engler, Die Ostdeutschen. Kunde von einem verlorenen Land (Berlin 1999); im folgenden zitiert: Engler, Die Ostdeutschen; Ina Merkel in ihrer Geschichte der Konsumkultur in der DDR „Utopie und Bedürfnis" (Köln, Weimar, Wien 1999), der von Evamaria Badstübner hrsg. Sammelband „Befremdlich anders. Leben in der DDR“ (Berlin 2000) oder das Buch über Punk, New Wave, HipHop und die Independentszene in der DDR in den 1980er Jahren: "Wir wollen immer artig sein ...4, hrsg. v. Roland Galenza, Heinz Havemeister (Berlin 1999).

24 Wie es z.B. Stefan Wolle in "Die heile Welt der Diktatur“ (Berlin 1998) für die 1970er und 1980 er Jahre der DDR gelungen ist; im folgenden zitiert: Wolle, Heile Welt. 
Günter Gaus einmal idyllisierend genannt hat ${ }^{25}$ - nicht bestehen, weil sie ihre Leute auf Dauer klein halten wollte; zugleich war sie aber ohne deren Engagement für das Land nicht lebensfähig. Der Bruch mit der eigenen Bevölkerung war von Beginn an im politischen System der DDR angelegt: ein Bruch, der sich in mehreren Phasen vollzog und der sich zugleich in besonderer Weise an der Generationenfolge festmachen läßt.

Dies ist auch der Grund, warum generationsgeleitete Ansätze zunehmend in die soziologische wie historische Analyse der DDR Eingang finden. Deren Modelle basieren dabei auf z.T. divergierenden Quellenlagen und sind von sehr unterschiedlicher Reichweite. Vier davon seien hier kurz erörtert und auf ihre Deutungsfähigkeit hinterfragt:

Die Generationsmodelle der Westberliner Autoren Dieter Geulen und Albrecht Göschel26 beruhen beide auf biographischen Interviews mit einer überschaubaren Anzahl an Gesprächspartnern ${ }^{27}$. Der Erziehungswissenschaftler wie der Soziologe entwerfen eine jeweils eigene Charakteristik der DDR-Generationen, wobei es sich bei beiden eher um eine Einteilung nach Jahrgangskohorten handelt: Geulen beschreibt in seinem Modell hauptsächlich drei Kohorten - die um 1940, 1950 und 1960 Geborenen. Die (jüngste) Kohorte, die der um $1970 \mathrm{Ge}$ borenen, spielt bei ihm dagegen kaum eine Rolle 28 . Auch Göschel unterteilt sein Modell, obwohl er ausdrücklich nicht von Kohorten, sondern von "historischen Generationen “29 spricht, lediglich nach Geburtsjahrgängen, wobei er der 40er-, 50 er- und 60 er-Jahre-Generation als vierte noch die 30 er-Jahre-Generation voranstellt. Das mit dieser rein schematischen Einteilung nach Geburtsjahrzehnten wesentliche gesellschaftliche Zäsuren in der Geschichte der DDR unberücksichtigt bleiben, ist offensichtlich ${ }^{30}$. Welche Generation aber letztlich ihre Potenzen ausbilden und zu kollektiven Impulsen formen kann, hängt mit der gesellschaftlichen

25 Günter Gaus, Wo Deutschland liegt. Eine Ortsbestimmung (Hamburg 1983) 45.

${ }^{26}$ Dieter Geulen, Typische Sozialisationsverläufe in der DDR. Einige qualitative Befunde über vier Generationen, in: APuZ 43 (1993) 26/27, 37-44; ders., Politische Sozialisation in der DDR. Autobiographische Gruppengespräche mit Angehörigen der Intelligenz (Opladen 1998); im folgenden zitiert: Geulen, Sozialisationsverläufe bzw. Politische Sozialisation.

Albrecht Göschel, Kontrast und Parallele - kulturelle und politische Identitätsbildung ostdeutscher Generationen (Stuttgart, Berlin, Köln 1999); im folgenden zitiert: Göschel, Kontrast und Parallele.

${ }^{27}$ Geulen führte Gruppengespräche mit insgesamt 35 Vertretern der Intelligenz, darunter vielen ehemaligen SED-Mitgliedern. Göschel sprach bei seinen Interviews in den Neuen Ländern mit 22 Partnern, die allesamt den „Kulturmilieus“ der DDR (Künstler, Sozialwissenschaftler, Mitarbeiter des Kulturapparates etc.) entstammten. Er konstruiert seine ostdeutschen Generationsprofile aus Gesprächen mit zwei bis acht Personen je "Kohorte*.

${ }_{28}$ "Zu dieser ja noch sehr jungen Generation ergibt sich ... noch kein differenziertes Bild“, in: Geulen, Sozialisationsverläufe 44.

29 Göschel, Kontrast und Parallele 28.

30 Auch wenn wesentliche historische Zäsuren in der DDR-Geschichte zufällig am Ende bzw. Beginn eines Jahrzehntes eintraten: wie die Gründung der DDR - 1949, der Bau der Mauer - 1961 oder der Machtwechsel von Ulbricht zu Honecker - 1971. Selbst der Kollaps der DDR - 1989/90 - geschah am Ende bzw. Anfang eines Jahrzehnts. 
Dynamik zusammen, in die sie hineingeboren wird: Die potentielle Energie einer Generation bedarf stets „der auslösenden Kraft des gesellschaftlich-geistigen Prozesses" ${ }^{* 1}$, um sich entfalten und auf den soziotären Entwicklungsgang zurückwirken zu können.

Der Leipziger Historiker Hartmut Zwahr und der Ostberliner Kultursoziologe Wolfgang Engler ${ }^{32}$ beziehen in ihren Modellen die Vorgängergenerationen der eigentlichen DDR-Jugendgenerationen als grundlegende Ausgangsposition mit ein. Hartmut Zwahr beginnt mit den Geburtsjahrgängen 1880 bis 1909 und fährt dann in Zehn-Jahres-Schritten kohortenmäßig bis 1949 fort. Die nachfolgenden Jahrgänge 1950 bis 1988 - die „eigentlichen Kinder der Republik“33 - unterteilt er im Gegensatz zu allen anderen Autoren jedoch nicht weiter. Seine Charakterisierung der Nachkriegsgeneration fällt äußerst knapp aus. Unterschiede zwischen den „reinen“ DDR-Jahrgängen arbeitet Zwahr nicht heraus. Wolfgang Engler dagegen spricht ausdrücklich von drei „politischen " Generationen in der DDR. Die Grenzen in seinem Generationenmodell sind weniger fließend. Er unterteilt in die zwischen 1890 und 1910 geborenen "Alten“, die um 1930 geborenen Jungen " und in die zwischen 1945 und 1950 geborenen, ostdeutschen Achtundsechziger. Zur Beweisführung ihrer Ansätze bedienen sich $Z$ wahr wie Engler sehr unterschiedlicher Belege: Sie reichen von historischen Dokumenten über die schwankenden Zahlen der Fluchtbewegung gen Westen bei Zwahr bis zu Oral-history-Zeugnissen und der Analyse kritischer Kunstwerke aus der DDR, auf die sich vor allem Engler immer wieder stützt.

Zwischen den vier hier erörterten Ansätzen ${ }^{34}$ zeichnen sich trotz aller Unterschiedlichkeit in ihrer Herangehensweise jedoch auch inhaltliche Parallelen ab: Sie

31 Mannheim, Generationen 553.

32 Hartmut Zwahr, Umbruch durch Aufbruch: Die DDR auf dem Höhepunkt der Staatskrise 1989. Mit Fxkursen zu Ausreise und Flucht sowie einer ostdeutschen Generationenübersicht, in: Harnut Kaelble, Jürgen Kocka, Hartmut Zwabr, Sozialgeschichte der DDR (Stuttgart 1994) 426-465; im folgenden zitiert: Zwahr, Umbruch; sowie Engler, Die Ostdeutschen.

33 Zwabr, Umbruch 451.

34 Weitere, z.'T. jedoch nur punktuelle Beschreibungen finden sich bei dem Kunsthistoriker Günter Feist, in: Option Gegenwehr. Korrektive zur Kunstpolitik der DDR (Köln 1995) $41 \mathrm{f}$., bei dem Literaturwissenschaftler Wolfgang Emmerich, in: Kleine Literaturgeschichte der DDR (Leipzig 1996), bei dem Kulturhistoriker Dietrich Müblberg, in: Wann war 68 im Osten? Oder: Wer waren die 68er im Osten?, in: Berliner Blätter 18 (1999); im folgenden zitiert: Müblberg, $68 \mathrm{im}$ Osten, (nach Internetpräsentation: http://user.berlin.sireco.net/ dpo.muehlberg/680st.htm) und vor allem immer wieder bei Dorotbee Wierling (u.a. in: Die Jugend als innerer Feind. Konflikte in der Erziehungsdiktatur der sechziger Jahre, in: Kaelble, Kocka, Zwahr, Sozialgeschichte der DDR (Stuttgart 1994) 404 ff.; dies., Die Grenzen der Mobilisierung. Mädchen in der FDJ der sechziger Jahre, in: Christine Benninghaus, Kerstin Kohtz, „Sag mir wo die Mädchen sind..." Beiträge zur Geschlechtergeschichte der Jugend (Köln 1999) 103 ff.; dies., Opposition und Generation in Nachkriegsdeutschland. Die Herausforderung der Nachkriegsgeborenen in Ost und West, in: Geteilte Vergangenheit - eine Geschichte? (Potsdam 1998, Manuskript); im folgenden zitiert: Wierling, Opposition und Generation. Dorothee Wierling setzt sich seit längerem dezidiert mit den Prägungen der „Generation der Nachkriegskinder" ${ }^{\prime}$ in der DDR auseinander. 
eint die Feststellung, daß politisches Bewußtsein und Handlungspotenzial in Abhängigkeit von den im Jugendalter gegebenen Sozialisationsbedingungen steht. Auch führen alle Autoren das Scheitern der DDR auf einen engen Zusammenhang von ökonomischen und gesellschaftspolitischen Konflikten und Diskursen zurück. Von entscheidender Bedeutung war dabei der ökonomische Faktor „Arbeit“. Nach dem Krieg habe er auf Grund des gemeinsamen Aufbauerlebnisses bindende Wirkung entfaltet, später jedoch wegen mangelnder Modernisierung in der Produktion zu Verdruß geführt ${ }^{35}$. Ebenso hätten staatliche Repression und innere Zerrissenheit reformwillige und artikulationsfähige politische Teilsysteme (Göschel) handlungsunfähig gemacht. Engler beschreibt diesbezüglich die spätere Spaltung der Ost-68er in "Politiker" und "Ethiker". Während erstere Reformen innerhalb des Systems durchsetzen wollten, hatten die Ethiker eher das Ziel, das SED-System durch gezielte Aktionen "von außen“ zu verunsichern ${ }^{36}$.

Im Zusammenhang mit den Konfliktlinien zwischen den Generationen sind auch die unterschiedlichen kulturellen Bezugspunkte der Autoren zu sehen. Benennt Göschel z.B. Differenzen zwischen der "Weimarer Generation" und den Geburtsjahrgängen vom Ende der 1920er bis Anfang der 1930er Jahre um die "Erstürmung der Höhen bürgerlicher Kultur “, beschreibt Engler, wie "die Jungen“ insbesondere im Bereich der (Film)Kunst von Funktionären reglementiert worden sind. Die Autoren erörtern auch Folgen der DDR-spezifischen Sozialisationsbedingungen für die Wende- und Nachwendezeit: Massenflucht in die Bundesrepublik, rechtsradikale Tendenzen, Mißtrauen in Institutionen, anhaltende Vorurteile gegen den Westen. Alle aufgezeigten Verhaltensweisen der Ostdeutschen werden von den Verfassern sozialisatorisch begründet. Sie durchbrechen damit, was ihnen als Verdienst anzurechnen ist, stereotype Vorstellungen vom Leben in der DDR. Problematisch ist jedoch, daß die unterschiedlichen Generationsentwürfe (insbesondere bei $Z$ wahr und Engler) kaum als durchlaufende Modelle dargestellt werden. So geht Engler in seiner Analyse der Ostdeutschen immer wieder auf deren generative Prägung ein, wendet seine Begrifflichkeiten allerdings bereits analysierend an, noch bevor er ein Gesamtkonzept vorgestellt hat. Auch werden durch ihn keine weiteren Interpretationsmöglichkeiten seines Ansatzes erörtert, obwohl dies dringend geboten erscheint, offenbaren sich dem Leser doch schnell Differenzen zwischen Englers Generationenmodell und seinem Gesellschaftsbild. Während er sein Generationenmodell vor allem über die Intelligenz definiert (was soziologisch Sinn macht, weil überwiegend sie die geistige Führerschaft in der Gesellschaft übernimmt und somit die öffentliche Wahrnehmung von Generationen prägt), betont er in seinem Gesellschaftsbild von der DDR die starke soziale und kulturelle Stellung der Arbeiterschaft. In seinem Generationenmodell kommt diese dagegen so gut wie nicht vor. Dort werden hauptsächlich die "Kulturschaffenden" als gestaltende Kraft vorgeführt. Die Arbeiter bleiben bei dieser Betrachtung stets außen vor. Lediglich bezogen auf $1968 \mathrm{kommt}$

35 Zwahr, Umbruch $452 \mathrm{f}$.

${ }^{36}$ Engler, Die Ostdeutschen $330 \mathrm{f}$. 
Engler kurz auf jene jungen Arbeiter zu sprechen, die das Gros der Protestierenden gegen die blutige Niederwerfung des „Prager Frühlings “ stellten. Er vermeidet also, sein Gesellschaftsbild konkret mit dem Generationenmodell zu verbinden. Die "Arbeiterklasse“ scheint in Englers Sicht über Generationen immer dieselbe geblieben zu sein. Bei ihr werden kein Wandel und keine Generationenbrüche festgestellt. Warum es aber gerade diese Gruppe war, die 1989 mehrheitlich auf die Straßen ging, bleibt bei Engler letztlich ungeklärt. Auch die starke Bedeutung der Arbeitsbindung in der DDR-Gesellschaft, wie sie Zwahr in seinem Ansatz erläutert, wird bei Engler kaum erwähnt. Dies ist erstaunlich, weil er gleichzeitig von der DDR als von einer im Kern „arbeiterlichen Gesellschaft ${ }^{\text {“ } 37}$ spricht. Diese Differenz macht deutlich, daß Engler idealtypische Generationsbilder aufbaut, indem er durchgängig von politisch aktiven, am Sozialismus orientierten Jahrgängen ausgeht. Selbst die antagonistischen Kräfte, die er in jeder Generation ausmacht, haben alle das gleiche Ziel: eine bessere DDR. Wirkliche Systemgegner (wie etwa in den 50er Jahren die Werdauer und Altenburger Widerstandsgruppen aus Schülern, Lehrlingen, jungen Arbeitern und Lehrern oder der Eisenberger Kreis u.a.) ${ }^{38}$ finden sich bei Engler in keiner Phase seiner DDR-Geschichte. So wird aber auch die zunehmende Distanz Jugendlicher zu diesem Gesellschaftssystem in den 1970er und 1980er Jahren nicht erklärbar.

\section{Politische Rahmenbedingungen generativer Entwicklung in der SBZ/DDR}

Die Geschichte der DDR aus der Perspektive ihrer Jugendgenerationen zu erzählen, macht schon deshalb Sinn, weil die Jugend in diesem Staat durchgehend eine zentrale Bezugsgröße gesellschaftlicher Planung war. Die SED-Führung hat vom Beginn ihrer Machtübernahme an auf die Jugend als Träger des Aufbaus der „neuen Gesellschaft ${ }^{*}$ gesetzt. Doch was ihr am Anfang noch weitgehend gelang, geriet ihr in den nachfolgenden Jahrzehnten immer stärker zum Desaster: Die Jugend war die erste Generation, welche die SED für sich vereinnahmen konnte, und sie war zugleich auch die erste, die ihr aus dem Ruder lief!

Dies unter Rückgriff auf den Generationenansatz zu beschreiben, vermag Entscheidendes zur Erhellung wichtiger Kontinuitäten und Diskontinuitäten in der Entwicklung der Jugend der DDR, aber auch des Landes selbst beizutragen. Es geht nicht darum, der in der Wissenschaft wie im Feuilleton grassierenden "Generationsetikettierungswut “39 neue Etiketten hinzuzufügen, diesmal eben nur für die Jugend-Ost, sondern mit Hilfe eines differenzierten Generationenansatzes tie-

37 Ebd. $197 \mathrm{f}$.

38 Vgl. dazu Einsichten 71-74, sowie Ehrhart Neubert, Geschichte der Opposition in der DDR 1949-1989 (Bonn 1997) 128-131.

39 Martin Kobli, Marc Szydlik (Hrsg.), Generationen in Familie und Gesellschaft (Opladen 2000) 7 . 
fer in den Zusammenhang von gesellschaftlicher und individueller Entwicklung in der DDR-Geschichte einzudringen: eine Geschichte, die, gemessen an ihrer Dauer, durch erstaunlich viele Brüche gekennzeichnet war, selbst wenn die DDR den darin lebenden und handelnden Menschen eher als ein politisches System von besonders zäher Konsistenz erschienen ist. Doch war es auch hier immer wieder das Privileg der Jugend, „die Verhältnisse zum Tanzen“ zu bringen. Die Frage, ob und in welchem Umfang dies den unterschiedlichen Jugendgenerationen der DDR gelungen ist, kann nur im Kontext jener entscheidenden Zäsuren in den 45 Jahren SBZ/DDR beantwortet werden, die „genau jene Weichenstellungen ausmachen, nach denen bestimmte Entwicklungen nicht mehr revidierbar ${ }^{40}$ waren. Das ist zugleich eng mit der Frage verbunden, ob die registrierten Einschnitte in die ostdeutsche Nachkriegsgeschichte zugleich auch Auslöser von Generationsbrüchen waren.

Die Mehrzahl der vorliegenden Periodisierungsversuche der DDR-Geschichte entstammen politikgeschichtlichen Analysen. Doch muß die vordergründige Heraushebung politischer Eckdaten als Zäsuren der Geschichte nicht a priori zu einem Gegensatz von „Herrschaft“ und „Gesellschaft" führen, vor allem dann, wenn verdeutlicht wird, daß den Individuen auch unter der Herrschaft von Diktaturen stets abweichende Reaktionen möglich sind. Es ist zu konstatieren, „in welchen Kräftefeldern von wem (und wie) Fremd- und Selbstkontrolle, Übermächtigung und Distanz angemeldet und eingefordert, versagt oder verwirklicht “ wurden. Damit werden „die Formen des unvermittelten Nebeneinanders wie der Verflechtungen von Repression und,Lockerung', von Mitmachen wie von Eigensinn, womöglich auch Widerständigkeit ${ }^{411}$ zum Thema der Analyse.

Die Geschichte von SBZ und DDR war kein monolithischer Block, kein über 45 Jahre ungebrochen durchlaufendes Band. Für ihre Periodisierung bieten sich unterschiedliche Zäsuren an. Die Zahl der von Zeitgeschichtsforschern veranschlagten Etappen schwankt zwischen drei und zehn, wobei sie oft in dem Maße anzusteigen scheint, je spezieller das Untersuchungsfeld gewählt wird. Überblicksgeschichten, wie die von Hermann Weber oder Ulrich Mählert, kommen in der Regel mit ca. fünf Etappen aus ${ }^{42}$.

In der Regel wird

- der Vorgeschichte vom Kriegsende im Mai 1945 bis zur Gründung der DDR am 7. Oktober 1949 ein erstes, abgeschlossenes Kapitel zugewiesen;

- die 2. Etappe umfaßt die Entwicklung bis zum Mauerbau 1961;

40 Cbristoph Kle $\beta$ mann, Die doppelte Staatsgründung. Deutsche Geschichte 1945-1955 (Bonn 1986) 11 ; im folgenden zitiert: Kleßmann, Doppelte Staatsgründung.

${ }^{1}$ Alf Lüdtke, Die DDR als Geschichte. Zur Geschichtsschreibung über die DDR, in: APuZ B 36 (1998) 3.

${ }^{42}$ Hermann Weber, Die DDR 1945-1990 (München 1993), sowie Ulrich Mäblert, Kleine Geschichte der DDR (München 1998). Wobei Weber an anderer Stelle: DDR. Grundriß der Geschichte 1845-1990 (Hannover 1991) auch differenziertere Gliederungen bis zu acht Etappen vornimmt. 
- als 3. Etappe wird die Phase der inneren Festigung der DDR bis zum Machtwechsel von Ulbricht zu Honecker im Jahr 1971 gewertet;

- die 70er Jahre, in ihrem Auf und Ab zwischen Stabilität und wachsender wirtschaftlicher Krise, gelten als 4. Etappe; während

- der in den 80ern, durch die Krise des sozialistischen Weltsystems und der Unfähigkeit der SED-Führung, produktiv darauf zu reagieren, eingeleitete Niedergang der DDR deren S. und letzte Etappe bildet. Sie gipfelt in der demokratischen Revolution des Herbstes 1989 und der Selbstaufhebung des Staates durch seine Bürger.

Dieser Einteilung folgend, möchte ich dennoch - aus sozialpsychologischen und mentalitätsgeschichtlichen Erwägungen heraus - partiell einige Zäsuren anders setzen: Für die frühen Phasen der DDR-Geschichte ist augenfällig, daß sich Übergänge von einer Etappe zur nächsten auf den Tag genau festmachen lassen und dies nicht nur in der Historiographie, sondern auch im Bewußtsein der beteiligten Menschen: Nach dem 17. Juni 1953 oder dem 13. August 1961 war für Bürger der DDR nichts mehr so wie zuvor. Generationen(um)brüche sind am ehesten an solchen einschneidenden historischen Terminen festzumachen (vgl. Abbildung 1). Gleiches dagegen gilt m.E. für den Gründungstag der DDR, den 7. Oktober 1949, weniger. Hier wurde per Dekret ein Prozeß zum Abschluß gebracht, dessen politische Grundlegung (und die damit verbundene generative Prägung) bereits in den vier Jahren zuvor vollzogen worden war. Daß die damit sankionierte Entscheidung für eine Zweistaatlichkeit Deutschlands auf lange Zeit manifest sein würde, entschied sich jedoch nicht an diesem Tag, sondern erst mit dem Scheitern des Volksaufstandes am 17. Juni 1953 und vor allem mit dem Bau der Mauer am 13. August 1961. In den späten Perioden der DDR verlief der Übergang von einer zur nächsten Etappe dagegen eher diffus, zeitlich zum Teil über mehrere Jahre gestreckt. Selbstverständlich gab es in diesen Phasen Ereignisse von nachhaltiger Wirkung wie z.B. das 11. Plenum des ZK der SED zu Fragen der Kultur und Jugend vom Dezember 1965, den Einmarsch der sowjetischen Truppen am 21. August 1968 in Prag oder die Ausbürgerung Wolf Biermanns am 16. November 1976. Doch waren diese Ereignisse nicht für alle (jungen) Bürger des Landes in gleicher Weise relevant. Ihre Folgen wurden erst schritrweise erlebbar, ohne zugleich für alle Mitglieder der jeweiligen Jugendgeneration die gleiche einschneidende Bedeutung zu erhalten, zumal sich die Spielräume für ein unterschiedliches Reagieren solchen Ereignissen gegenüber in der zweiten Hälfte der DDR deutlich differenzierten. Mentalitätsgeschichtlich entscheidend für die Charakterisierung der jeweiligen Generationseinheiten bleibt, wie sie diese Räume in den unterschiedlichen Phasen der DDR-Gesellschaft für sich ausgeschritten und gestaltet haben. Wie ist sonst, bei allem Beharren der SED-Führung auf ihrem unteilbaren Machtanspruch, das „Paradox von Stabilität und Revolution in der $\mathrm{DDR}^{* 43}$ zu erklären? Ständig zwischen Dogmatismus

43 So der Untertitel der verdienstvollen Analyse von Sigrid Meuschel, Legitimation und Parteiherrschaft in der DDR (Frankfurt a.M. 1992). 


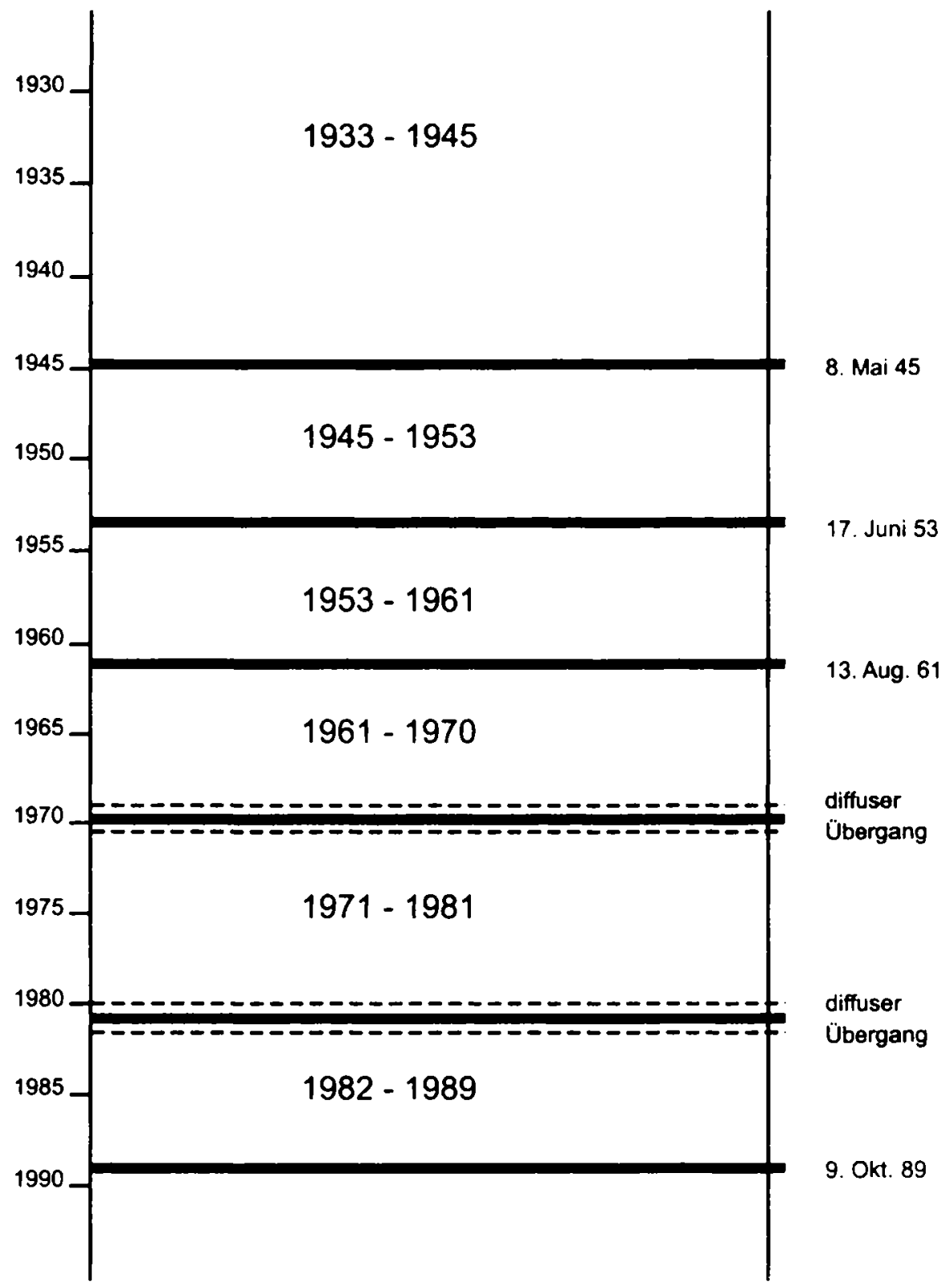

Abb. 1 
und (wirtschaftlich notwendigen) Modernisierungsversuchen schwankend, gelang es der SED-Führung nie, auf Dauer Mehrheiten der Bevölkerung für ihre Politik zu vereinnahmen. Immer wieder schlitterte sie in selbst herbeigeführte Krisen, die durch außenpolitische Entwicklungen oft noch verstärkt wurden. Die politische Sozialisation aller Jugendgenerationen der DDR wurde also in starkem $\mathrm{Maße}$ durch Krisenerfahrungen geprägt: Krisenerfahrungen, die partiell auch Generationsbrüche ausgelöst, zumindest aber mit herbeigeführt haben! Schon Mannheim verwies darauf, daß es "von der auslösenden Kraft des gesellschaftlich-geistigen Prozesses “ abhängt, „welche Generationslagerung in ihrer Potentialität aktiv wird“44.

Ein System von der politischen Stringenz der DDR prägte nachhaltig die L ebensformen der darin lebenden Jugendgenerationen, zumal deren ideologische Beeinflussung vordringlicher Auftrag aller staatlichen Instanzen und Institutionen - von den Schulen und Universitäten über die Kultureinrichtungen und die Medien bis hin zu den Parteien und Massenorgansiationen - war. Die Ausprägung gemeinsamer Generationszusammenhänge und markanter Generationseinheiten, sei es in Akzeptanz oder in Gegenwehr zu den staatlichen Vereinnahmungsversuchen, wurde dadurch zweifelsohne noch forciert.

Zugleich waren Jugendliche in der DDR nie "allein“ mit diesem politischen System: Die territoriale Nachbarschaft zur Bundesrepublik wie das Verbundensein mit ihr in einer gemeinsamen deutschen Geschichte waren als prägende Bezugsgrößen die gesamten vierzig Jahre über im Bewußtsein der Jugendlichen präsent - sei es aus eigenem Erleben wie vor dem Mauerbau oder über die "Westmedien“ danach. Diese Einflußnahme und Durchdringung gilt es stets mit im Blick zu behalten.

\section{Generationszusammenhänge in der DDR}

Im folgenden sollen die eingangs benannten drei Jugendgenerationen der DDR näher charakterisiert und zu dem Zeitachsenmodell (Abb. 1) ins Verhältnis gesetzt werden. Dabei ist zu beachten, daß sich diese Generationsgestalten nicht parallel, sondern quer zu zentralen zeitgeschichtlichen Etappen entwickelten (Abb. 2). Als init dem Ende des Zweiten Weltkriegs in der SBZ der "Weg zur Aufrichtung eines antifaschistischen, demokratischen Regimes, einer parlamentarisch-demokratischen Republik mit allen Rechten und Freiheiten für das Volk "45 offen schien, waren es die um 1930 Geborenen, die als erste Kohorte neu in das Jugendalter ${ }^{46}$ eintraten.

44 Mannheim, Generationen 553.

45 Aufruf des ZK der KPD an das deutsche Volk zum Aufbau eines antifaschistisch-demokratischen Deutschlands vom 11. Juni 1945, zitiert nach Kleßmann, Doppelte Staatsgründung 413.

46 Das Jugendgesetz der DDR vom 28. 1.1974 legt das Jugendalter ,auf die Zeit von 14 bis 25

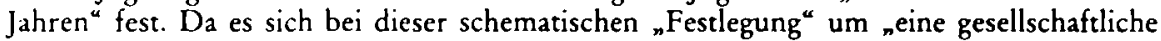




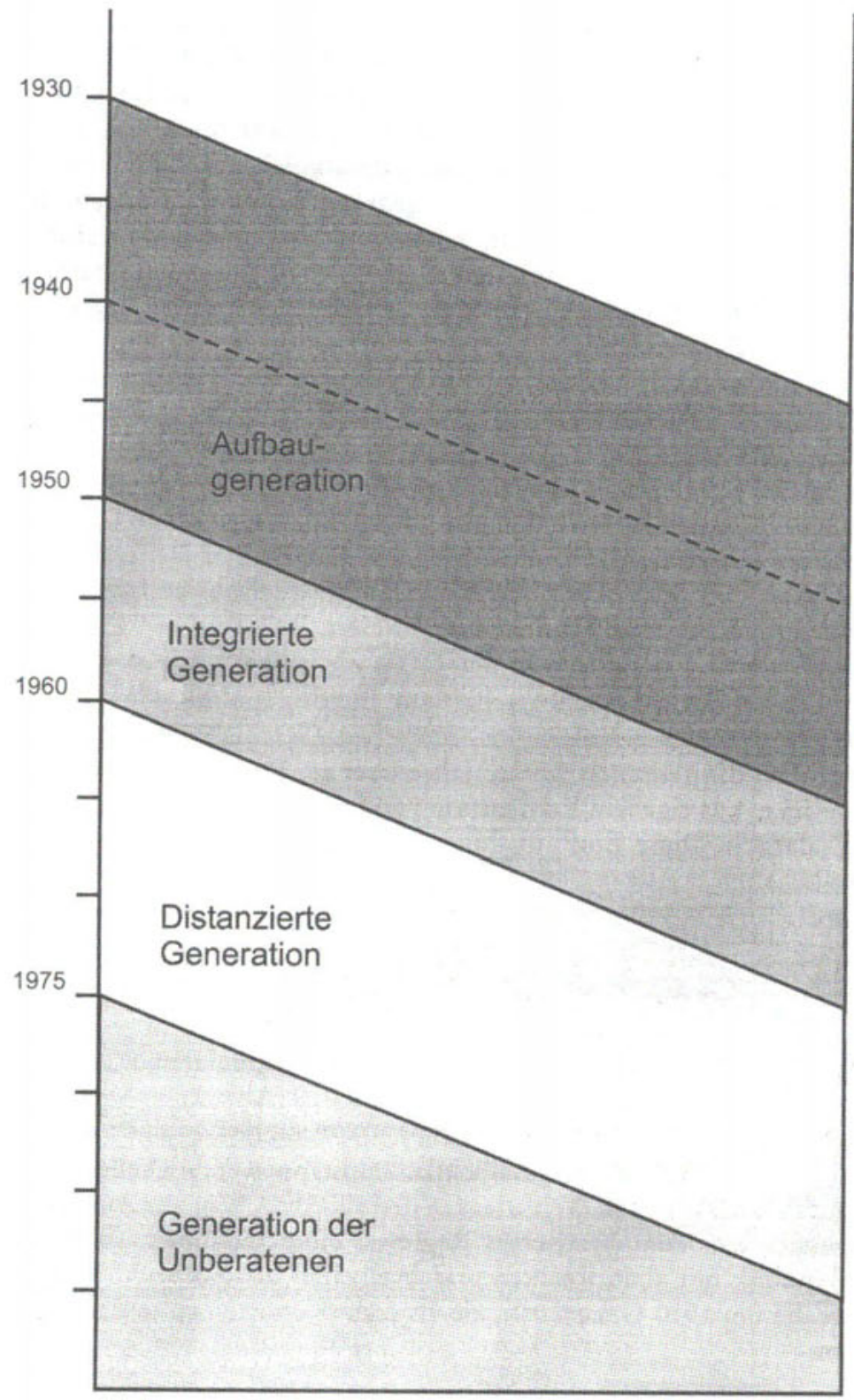

Abb. 2 


\section{Die Aufbaugeneration}

Der Beginn der Generationen(ab)folge der ostdeutschen Jugend ist also eindeutig mit dem Mai 1945 verbunden, ohne daß hier von einer "Stunde Null“ die Rede sein könnte. Jene Heranwachsenden, die um 1930 bis ca. 1940 geboren und demzufolge zwischen dem Kriegsende und Mitte der SOer Jahre ins Jugendalter hineinwuchsen, sind von zwei Gesellschaftssystemen wesentlich geprägt worden:

- In ihrer Kindheit durch den Nationalsozialismus und seinen ideologisch-totalitären Erziehungsapparat ${ }^{47}$, einschließlich der (traumatischen) Kriegserlebnisse an der "Heimatfront", wie partiell auch auf der Flucht oder bei der Vertreibung aus vertrauten Lebenswelten;

- in ihrer Jugend durch die materielle und psychische Not der Nachkriegszeit wie auch durch die (weitgehend) von Hoffnungen begleitete Aufbauphase in SBZ und DDR.

Entscheidung" der DDR-Gesetzgebung (in: Wörterbuch Jugendpolitik 103) handelt, die biologisch-akzelerative wie soziale Veränderungen und Entwicklungsbeschleunigungen im Jugendalter in der zweiten Hälfte des 20 . Jahrhunderts nur bedingt berücksichtigte, soll im folgenden die zeitliche Festschreibung des Jugendalters nach unten und oben flexibler gehandhabt werden.

So wurden z. B. im Jahr 1929 viele für die Aufbaugeneration der DDR wichtige Akteure geboren, die in dieser Zeit und danach das Land im positiven wie auch negativen Sinne mit geprägt haben; darunter FDJ-/SED-Funktionäre und (spätere) Minister wie Hans-Joachim Böhme, Johannes Chemnitzer, Hans-Joachim Hoffmann, Wolfgang Junker, Heinz Kuhrig, Werner Lamberz, Paul Markowski, Günter Schabowski, Kurt Turba; erstaunlich viele leitende Führungskader des Ministeriums für Staatssicherheit und der Nationalen Volksarmee wie Horst Felber, Joachim Goldbach, Werner Großmann, Manfred Hummitzsch, Werner Korth, Gerhard Neiber, Werner Prosetzky, Werner Rothe, Rolf Wagenbreth; (spätere) Kombinatsdirektoren und Werkleiter wie Werner Frohn, Wolfgang Gress, Klaus Henkes, Otto König, Herbert Kroker, Günter Pötschke - wichtige Wissenschaftler und (spätere) Institutsleiter wie Günter Feist, Walter Friedrich, Horst Haase, Karlheinz Lohs, Lutz Maier, Hansgünter Meyer, Gerhart Neuner, Heinrich Opitz, Helmut Seidel, Harald Thomasius, Hans Wagner, Wolfgang Weichelt; bekannte und erfolgreiche Künstler wie Kurt Böwe, Heiner Carow, Karl-Heinz Jakobs, Günter Kunert, Heiner Müller, Werner Tübke, Manfred Wekwerth, Christa Wolf. Vgl. Helmut Müller-Ensberg, Jan Wielgohs, Dieter Hoffmann (Hrsg.), Wer war wer in der DDR? (Bonn 2000).

Bei einer allzu mechanischen Handhabung der Generationsgrenzen würden all die hier genannten und für den Verlauf der DDR-Geschichte bedeutenden Personen, die ihre wichtigsten Prägungen in den ersten Jahren nach dem Zweiten Weltkrieg erhielten, fälschlicherweise keine Berücksichrigung in der Beschreibung der Aufbaugeneration finden.

47 Erika Mann schrieb bereits 1938 im Exil: „Keine Menschengruppe ... wurde so sehr, so entscheidend erfaßt von den Wandlungen, weelche die Nazi-Diktatur im Leben ihrer Untertanen vornahm, wie die Kinder. Denn während der erwachsene Deutsche zwar erstens Nationalsozialist zu sein hat, zweitens aber doch vorläufig noch Ladenbesitzer oder Fabrikant sein mag ..., ist das deutsche Kind schon heute ein Nazi-Kind und nichts weiter. Die Schule, die es besucht, ist eine $\mathrm{Nazi}$-Schule, die Jugendorganisation, der es angehört, ist eine $\mathrm{Nazi}$ Organisation, die Filme, zu denen man es zuläßt, sind Nazi-Filme, und sein Leben gehört ohne Vorbehalt dem Nazistaat “; Erika Mann, Zehn Millionen Kinder. Die Erziehung der Jugend im Dritten Reich ([Ost-]Berlin 1988) 19. 
Zahlenmäßig verstärkt wurde die Aufbaugeneration durch jene zwischen 1925 und 1929 geborenen Jahrgänge der sogenannten „Flakhelfer-Generation", deren männliche Angehörige überwiegend gegen Kriegsende noch in die Kampfhandlungen verstrickt worden waren. Obwohl dies den meisten der nach 1930 geborenen Jugendlichen erspart blieb, vereinte sie mit ihrer Vorgängergeneration eine emotional vergleichbare Ausgangslage. Aus der mit dem Zusammenbruch des Dritten Reiches desaströs endenden Vorgeschichte ihrer Jugend heraus, gab es "viele Gründe, völlig neu zu beginnen oder doch den Versuch dazu zu wagen ${ }^{4}+8$. In dieser Generation mischten sich, auf dem Hintergrund der nun allen offenbar werdenden Verbrechen des Nationalsozialismus, "übersteigertes Schuldgefühl“ und ein "kräftiger Schuß Verdrängungssehnsucht ${ }^{49}$ zu einer neuen Aufbruchstimmung.

Dem kam die politische Realität in der SBZ entgegen. Forderten die antifaschistisch-demokratischen Parteien anfangs noch individuelle Schuldbekenntnisse und die Bewährung jedes Einzelnen, hieß es bereits wenig später - diktiert vom Bemühen, die gesamte Jugend erneut politisch zu mobilisieren -, daß sich nun auch „ehemalige einfache Mitglieder und untere unbesoldete Funktionäre in der HJ und im BDM (...) in die Schar der aktiv am Aufbau Beteiligten " 50 ohne Vorbedingungen einreihen könnten.

An der Glaubwürdigkeit dieser Offerte gab es anfangs bei den wenigsten Jugendlichen Zweifel. Erst mit der immer deutlicher werdenden Intoleranz der SED- wie der FDJ-Führung gegenüber Andersdenkenden und deren zunehmend direkter vorgetragenen Alleinvertretungsansprüchen kamen bei einer wachsenden Zahl von Jugendlichen $Z$ weifel auf. Doch kollidierte dieser stets mit dem eigenen schlechten Gewissen:

„Denn die eifernde Schulleiterin hatte unter Hitler im Gefängnis gesessen, der dogmatische und gebildetste der Dozenten war ein Emigrant gewesen - man selbst aber hatte Hitler gedient. Man war ... in moralischer Hinsicht der Schwächere ... Die Versuchung war groß, der Vernunft abzuschwören, um ehrlichen Herzens in der Glaubensgemeinschaft aufgehen zu können. Es waren nicht die Schlechtesten, die diesen Weg wählten, der alles andere war als bequem. ${ }^{451}$

\section{Zwabr, Umbruch 449.}

49 Engler, Die Ostdeutschen 321 bzw. 17. Der Soziologe Hansgünter Meyer, zu Kriegsende sechzehn Jahre alt, brachte dieses Lebensgefühl vieler seiner Altersgenossen später in seinen Lebenserinnerungen auf den Punkt: „Ich fühlte mich im Nachhinein auf furchtbare Weise betrogen. In meinem Kopf bildete sich immer wieder der gleiche Satz: Das zahlt Ihr mir heim!“, ders., Die Entdeckung der Soziologie. Eine intellektuelle kolumbianische Erfahrung, in: Christian Fleck (Hrsg.), Wege zur Soziologie nach 1945. Autobiographische Notizen (Opladen 1996) 269.

50 Offene Tore für die Jugend, in: Deutsche Volkszeitung, Zentralorgan der KPD, vom 27. 9. 1945. Vgl. dazu auch Ulrich Mählert, Gerd-Rüdiger Stephan, Blaue Hemden, rote Fahnen. Die Geschichte der Freien Deutschen Jugend (Opladen 1996) 25-28; im folgenden zitiert: Mählert, Stephan, Blaue Hemden.

51 Wie sich der werdende Neulehrer Günter de Bruyn (Jahrgang 1926) später erinnert, in: Zwischenbilanz. Eine Jugend in Berlin (Frankfurt a.M. 1992) $374 \mathrm{f}$. 
Dies war ein Weg, der vielen von ihnen zugleich einen deutlichen sozialen Aufstieg bescherte. Sie profitierten von dem zur Zerschlagung des Nationalsozialismus in den Köpfen notwendig gewordenen Elitenaustausch. Junge, überwiegend proletarische Aufsteiger wurden so zu einer tragenden Säule der neuen Gesellschaft. „Sic waren (Aus-)Gestalter eines gesellschaftlichen Systems, für das es noch keinen erprobten Bauplan gab. ${ }^{~} 52$ Den Ton jedoch gaben andere an: die "Alte Garde“ der zwischen 1880 und 1914 geborenen „Gründergeneration ${ }^{\text {" des }}$ SED-Staates ${ }^{53}$, die - zur Tragik aller Jugendgenerationen der DDR - die politische Macht bis zum selbst verschuldeten Ende nie mehr aus den Händen geben sollte auch um den Preis, die Jugend schrittweise zu verlieren! In den Anfangsjahren jedoch führte für die aufstiegswilligen Jugendlichen im Osten noch kein Weg an den „politischen Alten“ vorbei: „Hier war der Aufbau ein Ausstieg aus Unterschichten durch Staatsdienst, weil Staat und Partei die universellen Arbeitgeber waren. ${ }^{\text {"5 }}$

Die ersten Jahrgänge der Aufbaugeneration haben als Jugendliche die Entwicklung der DDR zur Einparteiendiktatur in vollem Umfang miterlebt. Im Juni 1953 waren sie dem Jugendalter fast schon entwachsen, während sich die Mitglieder der jüngsten Kohorte dieser Generation, die um 1940 Geborenen, zu diesem Zeitpunkt gerade anschickten, in das Jugendalter hineinzuwachsen. Am Ende ihrer Jugendzeit stand die Mauer bereits. Je nach den konkreten Lebensumständen ihres Aufwachsens und deren unterschiedlicher individueller Verarbeitung führte das bei den beteiligten Jugendlichen - in ihrer Mehrheit - entweder zu einer relativ stabilen Verankerung in dieser Aufbaugeneration oder - bei einer Minderheit - zu einem frühen Ausstieg aus der DDR-Gesellschaft durch Flucht in den Westen oder den Rückzug in die innere Emigration. Von 1949 bis zum 13. August 1961

52 Demzufolge erinnern heute die sozialen Aufsteiger jener Zeit die 50er als ihre besten Jahre. Vgl. Thomas Abbe, Michael Hofmann: „Eigentlich unsere beste Zeit." Erinnerungen an den DDR-Alltag in verschiedenen Milieus, in: APuZ B 17 (2002) 20.

${ }^{53}$ Engler (Die Ostdeutschen 320) weist zu Recht daraufhin, daß ihre „Erfahrungen im Klassenkampf der späten Weimarer Republik ... derart einschneidend und existentiell prägend (waren), daß sie die große altersmäßige Streuung “ dieser politischen Generation aufgewogen haben.

${ }^{54}$ Müblberg, 68 im Osten 8. Diese Entwicklung wurde in der zweiten Hälfte der DDR partiell dadurch unterlaufen, daß sich die Intelligenz in der DDR zunehmend aus sich selbst reproduzierte. Kinder aus unteren Schichten fanden immer weniger Zugang zur akademischen Bildung; der Elitentausch war historisch abgeschlossen. Vgl. Gustav-Wilhelm Bathke, Kurt Starke, Studentenforschung, in: Walter Friedrich u. a. (Hrsg.), Das Zentralinstitut für Jugendforschung Leipzig 1966-1990. Geschichte, Methoden, Erkenntnisse (Berlin 1999); im folgenden zitiert: Friedrich, Das Zentralinstitut.

Politisches Wohlverhalten gegenüber der „Diktatur der Alten“ war aber auch später weiterhin Voraussetzung, wenn man im engeren Apparat von Staat und Partei sowie den angelagerien ideologischen Dienstleistern (wie Medien, Schulen, Universitäts- und Akademieleitungen) und den "Chefetagen" der Wirtschaft aufsteigen wollte, während in Technik, Naturwissenschaft und auch weiten Teilen der anderen Forschungsrichtungen immer stärker das Spezialistentum sich durchsetzen konnte. 
verließen ca. 2,7 Millionen Menschen die DDR ${ }^{55}$, ,in der zweiten Hälfte der fünfziger Jahre vorzugsweise aus der jungen Generation, die sich einen Neuanfang ,drüben' zutraute" 56 .

Zeitgenössische westdeutsche Quellen belegen zugleich, wie nachhaltig die den ostdeutschen Jugendlichen gewährten Bildungschancen auf deren Verbleib in der DDR bzw. auf ihr ideologisches Wohlverhalten darin wirkten. Von den 400000 Absolventen der Diplomjahrgänge 1952 bis 1963 sind nach Schätzungen von Ernst Richert höchstens 3,8 Prozent in den Westen abgewandert. Nur 15 Prozent von ihnen wurden als Gegner des Systems vermutet. Aber als engagierte Parteigänger des SED-Staates - sei es als überzeugte Aktivisten (5 Prozent) oder als "opportunistische Karrieristen“ (10 Prozent) - konnte von ihm auch nur eine Minderheit ausgemacht werden. Über zwei Drittel der Aufbaugeneration wären demzufolge lediglich als mit dem Sozialismus "Arrangierte" einzustufen ${ }^{57}$, was sich mit den Erkenntnissen aller heute zugänglichen Dokumente zum schwindenden Einfluß von FDJ und SED auf die DDR-Jugendlichen der 50er Jahre deckt ${ }^{58}$.

Ende oder Anfang? Gehen oder Bleiben? Die politischen und wirtschaftlichen Krisen jener Jahre wurden von den Jugendlichen der DDR noch als Alternativen zum Weiterbestehen des Staates bzw. einem individuellen Verbleiben darin erlebt. Daß im ersten Jahrzehnt der DDR dennoch die Aufbaugeneration und nicht der zum Ausstieg aus dem sozialistischen System neigende Teil der Heranwachsenden die Deutungshoheit über die Jugend des Landes erringen konnte, hat gerade im Verweigerungspotenzial der "Aussteiger" seine Ursache. Durch ihren Weggang in den Westen bzw. ihren Rückzug in die innere Emigration enthoben sie sich der Möglichkeit der direkten Einflußnahme. Dies geschah nicht immer freiwillig, gingen doch Partei, Staat und immer stärker auch die FDJ in den frühen Jahren der DDR gegen politisch nnicht paßfähige" Jugendliche, wie etwa die Mitglieder der evangelischen ${ }_{n} J u n g e n$ Gemeinde" oder die Jugendweihe-Verweigerer, mit besonderer Schärfe vor. Demzufolge haben wir es bei der Gegengruppe zur Aufbaugeneration mit einer im doppelten Sinne ausgegrenzten Generationseinheit zu tun.

55 Vgl. dazu: Der Fischer Weltalmanach. Sonderband DDR (Frankfurt a.M. 1990) 134, sowie Zwabr, Umbruch 438-447.

56 Zwabr, Umbruch 443. Der Anteil der 16- bis 25jährigen unter den "Republikflüchtlingen“ schwankt zwischen 17,9 Prozent im I. Quartal 1953 und 43,2 Prozent im IV. Quartal 1956. Der Anteil der Jugendlichen an der Gesamtbevölkerung betrug zum damaligen Zeitpunkt aber lediglich ca. 15,8 Prozent. Vgl. dazu auch Peter Skyba, Vom Hoffnungsträger zum Sicherheitsrisiko. Jugend in der DDR und Jugendpolitik der SED 1949-1961 (Köln, Weimar, Wien 2000) $304 \mathrm{ff}$; ; im folgenden zitiert: Skyba, Hoffnungsträger.

57 Emst Richert, Sozialistische Universität ([West-]Berlin 1967) 247.

58 .Zu keinem Zeitpunkt war es der SED-Führung gelungen, auch nur annähernd das gewünschte $\mathrm{Maß}$ an Loyalität unter den Heranwachsenden der DDR zu erzeugen (...) Sie scheiterte ... daran, die Masse der Jugendlichen über die formale Mitgliedschaft hinaus an den (Jugend)Verband zu binden“, Skyba, Hoffnungsträger 419. Vgl. auch Mählert, Stephan, Blaue Hemden 102-136. 


\section{Die Integrierte Generation}

Die Bereitschaft zur Einpassung in das gesellschaftliche System der DDR, auch wenn es innerlich nur bedingt akzeptiert wurde, konnte vor 1961 individuell sehr unterschiedlich begründet und motiviert sein: familiäre und lokale Bindungen, soziale Aufstiegschancen, Stolz auf das Geschaffene und Erreichte, geringe Flexibilität, politisches Desinteresse oder auch Vorbehalte gegen die westdeutsche Gesellschaft, die sie nicht als eine persönliche Alternative zur DDR erscheinen ließen. Nach dem Bau der Mauer waren all diese Gründe irrelevant geworden. Die SED-Führung hatte den noch Zögernden die Entscheidung aus der Hand genommen; nun war der DDR auf lange Zeit nur noch unter Riskierung des eigenen Lebens zu entkommen.

Doch das allein erklärt nicht, warum jener Teil der Jugendlichen, der sich zur DDR als persönlichem Lebensentwurf bekannte, in der Nachfolgegeneration weiterhin tonangebend blieb. Keine Jugendgeneration im kurzen Leben des zweiten deutschen Staates war integrierter darin als die um 1945 und bis 1960 Geborenen, die zwischen dem Mauerbau und Mitte der 1970 er Jabre ins Jugendalter hineinwuchsen ${ }^{59}$.

Und dies galt nicht nur für die aufstiegsorientierten Abiturienten und Studenten, wie erste soziologische Erhebungen belegten ${ }^{60}$. So herrschten , bereits Anfang der 60er Jahre bei der Mehrheit der 14- bis 18jährigen Schüler und Lehrlinge ... positive Einstellungen zu den propagierten sozialistischen Zielen und Werten vor. Charakteristisch war eine hohe Identifikation mit der DDR. “ Dies war eine Entwicklung, die sich in allen Gruppen und Schichten der Jugend bis weit in die 70er Jahre hinein konsolidieren sollte ${ }^{61}$.

Am Beginn ihrer Jugend erlebten sie - trotz Mauerbau und Abschottung gen Westen -- auch viele produktive Entwicklungen, ging doch gerade von der Jugendpolitik zwischen 1963 und 1965 „eine Tendenz zur Demokratisierung der Gesellschaft aus, die nicht im Bereich des Appells verblieb ${ }^{* 62}$. Erste Jugendclubs entstanden, der „Gitarrenbeat" wurde von der FDJ offiziell gefördert, und mit DT 64 erhielten die Jugendlichen aus Anlaß des Deutschlandtreffens 1964 sogar ein

59 Hier ist unbedingt darauf zu verweisen, daß sich die Jahrgangsgrenze zwischen der Aufbaugeneration und der Integrierten Generation - was das Ende der einen und den Beginn der anderen betrifft - entwicklungsbedingt leider nicht trennscharf bestimmten läßt. Beide Jugendgenerationen der SBZ/DDR gehen in den um 1945 geborenen Kohorten nahezu nahtlos ineinander über (vgl. Abb. 2).

6O In der DDR konnte sich die lange als „bürgerliche Wissenschaft" bekämpfte Soziologie erst ab Mitte der 1960er Jahre schrittweise etablieren. Für die Jugendforschung ist ihre Geschichte eng mit dem 1965 in Leipzig gegründeten und bis Ende 1990 dort tätigen ZIJ verbunden. Vgl. Friedrich, Das Zentralinstitut 13-69.

61 Peter Förster, Die Entwicklung des politischen Bewußtseins der DDR-Jugend zwischen 1966 und 1989, in: Friedrich, Das Zentralinstitut 78; im folgenden zitiert: Förster, Politisches Bewußtsein.

62 Leonore Krenzlin, Vom Jugendkornmuniqué zur Dichterschelte, in: Kahlschlag. Das 11. Plenum des ZK der SED 1965. Studien und Dokumente, hrsg. v. Günter Adge (Berlin 1991) 151. 
eigenes Radioprogramm. Dieser Faden wurde nach Jahren der Stagnation infolge des 11. Plenums des ZK der SED 1965, das insbesondere die Künste und die Jugendlichen erneut mit einschränkenden Verdikten belegte ${ }^{63}$, sowie nach dem Machtwechsel von Ulbricht zu Honecker - auch und gerade unter dem Druck der Jugendlichen - schrittweise wieder aufgenommen.

Trotz all der gegensätzlichen Entwicklungen blieb die Bindung des Gros dieser Jugendgeneration an die DDR während ihrer gesamten Zeit weitgehend stabil. Hauptursache dafür war, daß ihre Jugend in die Phase des relativen Wohlstands und der zunehmenden Anerkennung der DDR fiel (3. und 4. Zeitetappe, vgl. oben). Gleichzeitig wurde ihnen durch eine Reihe konkreter Angebote des Staates - vom Erlaß des weitreichenden Jugendgesetzes 1964 bis zu den Weltfestspielen der Jugend und Studenten 1973 in Ost-Berlin - ein Gefühl des Gebraucht- und Gefördertwerdens gegeben. Sie "waren die ersten Kinder der anderen Republik, die nichts als DDR erlebten " 64 . Fast alle von ihnen waren ohne allzu starke, innere Widersprüche Jungpioniere, gingen nahtlos in die FDJ über und feierten ihre Jugendweihe. Die älteren Kohorten unter ihnen legten in diesem Zeitraum bereits sogar das Abitur oder die Facharbeiterprüfung ab, manche beendeten schon ihr Studium. Als Gründer einer jungen Familie kamen sie nach 1971 bereits in den Genuß der großzügigen Förderung durch das unter Erich Honecker verkündete neue SED-Programm der "Einheit von Wirtschafts- und Sozialpolitik“ mit seinem zinslosen Ehekredit, dem Babyjahr und der bevorzugten Versorgung mit Kinderkrippenplätzen und Wohnraum. Alles Gute kam „von oben“: In der DDR war daher "der Topos der Dankbarkeit politisch stark instrumentalisiert "65, wie Dorothee Wierling ihren lebensgeschichtlichen Interviews mit Ostdeutschen der Jahrgänge 1949/50 entnimmt. Einer von ihnen erinnert sich: „Wir wurden doch als 49er in etwas hineingeboren, das wir selbst nicht mit entwickelt hatten. (...) Es war nicht die Zeit Bedingungen zu stellen. " 66 Aus all diesen Erfahrungen heraus glaubten viele von ihnen - trotz durchaus wahrgenommener Krisensymptome (CSSR 1968, Biermann-Ausbürgerung 1976) - lange an eine Reformierbarkeit der DDR, hatten sie doch diese in der Phase ihrer tatsächlichen Prosperität erlebt. Diese Generation hat „die politischen und insbesondere moralischen Ansprüche des Sozialismus als Ideologie besonders stark verinnerlicht "; ihre nemotionale Beziehung zur DDR als Heimat " war sehr stark ausgeprägt ${ }^{67}$. Ich nenne sie daher die Integrierte Generation.

Damit steht sie in einem direktem Widerspruch zu der aufbegehrenden Generation der $68 \mathrm{er}$ in den westlichen Industriestaaten. Als eingepasste, weitgehend systemkonforme Generation steht sie aber auch im Widerspruch zu einer Theorie,

${ }^{63} \mathrm{Vgl}$. dazu Bernd Lindner, Denkt bloß nicht, wir heulen. Das 11. Plenum des ZK der SED 1965, die DEFA und die Jugend, in: Deutschland Archiv 6 (2000) 891-901.

64 Andreas Molitor, Die Gruppe 49, in: Die Zeit 21 (20.5. 1999) 16; im folgenden zitiert: Molitor, Die Gruppe 49.

65 Wierling, Opposition und Generation.

66 Zitiert nach Molitor, Die Gruppe 49, 16.

67 Geulen, Sozialisationsverläufe 43. 
welche "die 68er-Generation als erste globale Generation "68 begreift, weil "die Studentenunruhen ... ebenfalls in Staaten des Ostblocks und der Dritten Welt eine Generationserfahrung darstellen, die bisher vernachlässigt ${ }^{\alpha}$ worden sind ${ }^{69}$.

Hat es „die 68er" in der DDR also nicht gegeben? Die Meinungen hierüber gehen weit auseinander: Dietrich Mühlberg kommt in seiner systemvergleichenden Ost-West-Analyse zu der Aussage, daß 1968 - wenn überhaupt - in der DDR eine (Zwischen-)Generation früher als im Westen aufgetreten ist hat: „Sie wäre als nachstalinistische Aufbaugeneration zu fassen, deren jugendliche Orientierungsphase mit einem enormen Modernisierungsschub" der End-50er und beginnenden 60er Jahre zusammenfiel. Sie sahen "seine sozialistische Bewältigung“ als ihre Aufgabe. "Freilich: eine Revolte haben sie nicht veranstaltet", ihre Ideale waren eher die wissenschaftlich-technische Revolution und die NÖ ${ }^{70}$. Doch zeigte sich in der DDR bald eine zunehmende Diskrepanz zwischen "objektiver" und "subjektiver" Modernisierung der Gesellschaft, die aber - anders als im Westen - nicht als Konflikt der Generationen ausgetragen wurde. Für Albrecht Göschel versäumte „die 40er-Jahre-Generation der DDR ${ }^{71}$ die emanzipatorische Dimension des Statuskonfliktes der 60er Jahre und bleibt sowohl den alten Eliten und Parteikadern untergeordnet als auch tradierten Pflicht- und Akzeptanzwerten verhaftet "72. Eine den West-68ern vergleichbare Generationseinheit kann die altersgleiche Kohorte im Osten, auch und vor allem unter dem repressiven Druck der in Prag aufzichenden Panzer ${ }^{73}$, nicht ausprägen. Das sieht Dorothee Wierling ähnlich, obwohl sie mit dem politischen Scheitern des Prager Frühlings „eine gemeinsame Lage in der Geschichte, einen Zusammenhang gemeinsamer Erfahrungen “

68 Beate Fietze, 1968 als Symbol der ersten globalen Generation, in: BJfS Band 7 (1997) 3, 367; im folgenden zitiert: Fietze, Globale Generation.

69 Fietze, Globale Generation 366. Ähnliche, wenn auch nicht so weitreichend ausformulierte Ansätze finden sich dazu u.a. bei: John R. Gilles, Geschichte der Jugend. Tradition und Wandel im Verhältnis der Altersgruppen und Generationen (Weinheim und Basel 1980) 187-222; Almuth und Klaus-Jürgen Bruder, Jugend. Psychologie einer Kultur (München, Wien, Baltimore 1984); Claus Leggewie, Die 89er. Portrait einer Generation (Hamburg 1995) 90-97, im folgenden zitiert: Leggewie, Die 89er. Heinz Bude (vgl. Beitrag im vorliegenden Band) hat dem zu Recht entgegengehalten, daß es sich bereits bei der Kriegsgeneration von 1914 um dic erste globalc Generation gehandelt hat und jede weitere ausgeprägte Generation des 20. Jahrhunderts zumindest europäisch war.

70 Mühlberg, 68 im Osten. NÖS oder auch NÖSPL - Neues Ökonomisches System der Planung und Leitung der Volkswirtschaft -, dessen Entwurf und Niedergang eng mit dem Schicksal des Vorsitzenden der Staatlichen Plankommission Erich Apel (geb. 1913) zusammenhing, der sich am 3.12. 1965 aus Protest gegen Ulbrichts Wirtschaftspolitik das Leben nahm.

71 Die aus der Sicht vieler westdeutscher Soziologen und Zeithistoriker in etwa den Kern der $68 \mathrm{er}$-Generation der Bundesrepublik bildeten. Vgl. Leggewie, Die 89er, 90, der die Jahrgänge 1940-1950 dazu zählt, während Bude sie bereits im Untertitel seines Generationsporträts (Bude, Altern) um zwei Jahre auf „die Jahrgänge 1938-1948“ vorverlegt.

72 Göschel, Kontrast und Parallele 158.

73 Nach der DDR 1953 sowie Ungarn und Polen 1956 stellten die sowjetischen Kommunisten 1968 in der CSSR unter Einsatz von massiver Waffengewalt binnen 15 Jahren zum 3. Mal klar, daß sie Abweichungen von ihrer politischen Doktrin im Ostblock nicht dulden wollten. 
zu den West-68ern ausmacht. Dennoch gab es ihrer Ansicht nach in der DDR "nicht jene fröhliche Selbststiftung als Generation wie im Westen “, weil die Protestierer dort isoliert und "ohne zusammenhängende geistige Orientierung “ blieben. „1968 wurde in der DDR zur Chiffre für eine Niederlage, nicht aber ein einheitsstiftendes Datum" 74 .

Demzufolge spricht Wierling für den Osten nicht von einer "Generation der 68er“, was Bude durchaus tur, auch wenn er dieses Datum für die DDR (analog zu Wierling) als „eine Geschichte der verlorenen Würde“ ansieht. Die Ost-68er hatten „das große Problem, daß sie ihren Takt in der Gesellschaft nicht durchsetzen konnten " 75 . Auch Engler sieht die Ost-68er in der Geschichte scheitern, und dies gleich zweimal: 1968 selbst und dann noch einmal im Herbst 1989, weil sie sich durch ihre in den 80er Jahren vollzogene Spaltung in sozialistische "Reformisten" und "idealistische Außenseiter" (gemeint sind damit die Keime der Bürgerbewegung) einander entfremdeten und sich so 1989/90 der Möglichkeit eines gemeinsamen Handelns beraubten. Das ${ }_{n}$ war für beide Fraktionen ein Unglück ${ }^{476}$. Anders die Ostberliner Psychologin Annette Simon: $Z$ war sieht auch sie, daß die 68er in der DDR nkeine kulturrevolutionären Veränderungen wie ihre westlichen GenerationsgenossInnen" auslösten; sie "wollten Reformen und setzten letztendlich eine Revolution in Gang, in der es zu einem Wechsel der gesellschaftlichen Produktionsverhältnisse gekommen ist". Aber: „Eigentlich sollte eine Generation auch daran gemessen werden, inwieweit sie der nächsten Generation Zukunft eröffnet oder verschließt. " $" 7$

Einmal abgesehen davon, daß man der Beschreibung einer möglichen Ost-68erGeneration nicht gerecht werden kann, wenn man sie wie Engler allein als eine "Bewegung" von Intellektuellen aus Kultur und Politik oder wie Simon unter dem Aspekt des Generationenkonflikts zwischen leitenden Funktionären des SED-Staates und ihren Kindern schildert ${ }^{78}$, ist es meines Erachtens auch wenig sinnvoll, aus einer 21 Jahre später nachgeholten Revolution den prägenden Charakter einer Generation für ihre Altersgleichen in der DDR im Jahre 1968 ableiten zu wollen. Die Wahrheit ist, daß die Ost-68er in ihrer Generation marginal blieben und bestenfalls als nausgebremste Generation“ (Bude) gelten können, während die Mehrheit der Jugendlichen sich in das System integrierte: $\ldots .$. lange Jahre war selbst in intellektuellen Kreisen der Prager Frühling kein Thema. Die Angst allein kann nicht der Grund dafür gewesen sein. Es war wohl auf eine schwer zu

74 Wierling, Opposition und Generation (Manuskript).

75 Von Machern und Halbstarken. Die Bundesrepublik und ihre Generationen. Interview mit dem Soziologen Heinz Bude, in: Die Zeit 21 (20.5. 1999) 14.

76 Engler, Die Ostdeutschen 328-338.

77 Annette Simon, Vor den Vätern sterben die Söhne? Die Achtundsechziger der DDR, in: Annette Simon, Jan Faktor, Fremd im eigenen Land? (Gießen 2000) $22 \mathrm{f}$.

78 Von den 506 von Staatssicherheit und DDR-Justiz 1968 eingeleiteten Ermittlungsverfahren gegen Jugendliche "wegen Verbreitung selbst gefertigter Hetzschriften " betrafen lediglich 7,6 Prozent Studenten, aber 71,1 Prozent junge Arbeiter und Lehrlinge. Vgl. Bernd Lindner, Enttäuschte Hoffnungen, in: ZFL, Einsichten 118-124 und Wierling, Opposition und Generation. 
erklärende Weise degoutant, über den Käfig zu reden, in dem man hockte. Ein miefiges Gefühl des Wohlseins und der Übereinstimmung mit dem System prägten die siebziger und frühen achtziger Jahre. ${ }^{49}$ Das Widerspruchspotenzial dieser Generation war nicht politisch, sondern vor allem kulturell geprägt.

\section{Die distanzierte Generation}

Die Ausformung prägender Generationseinheiten war in der DDR lange Zeit also auch eine Verdrängungsleistung! Dadurch ließen sich - so erstaunlich dies im Nachhinein anmutet - auch tiefe gesellschaftliche Einschnitte wie die Niederschlagung des Prager Fühlings 1968 oder die willkürliche Ausbürgerung Wolf Biermanns aus der DDR 1976 von den Jugendlichen mehrheitlich mental naussitzen“. "Die biographische Kontinuitätsnorwendigkeit (auch in bezug auf die Elterngenerationen - B.L.) oder der Zwang zur Konsistenzsicherung kann es verlangen, daß dramatische Veränderungen in der Umwelt weggeschoben werden. Selbst politische Kollektivereignisse werden gar nicht wahr- oder erst nach einer gewissen Zeit zur Kenntnis genommen. ${ }^{\text {"80 }}$

Erst eine neue Jugendgeneration - zwischen 1961 und 1975 geboren, von Mitte der 1970er Jahre bis zum Ende der DDR ins Jugendalter hineingewachsen - war dazu fähig, sich innerlich aus den alten $Z$ wängen zu befreien: Nachgeborene, für die der schmale Wohlstand der DDR nicht mehr eine historische Errungenschaft, sondern etwas Vorgefundenes und selbstverständlich in Anspruch Genommenes war. So vermochten sie auch eher dessen zunehmende Brüchigkeit zu erkennen und den dafür zu entrichtenden Preis an politischer Anpassung - den ihre Elternund Vorgängergeneration zu großen Teilen noch zu zahlen bereit war - in Frage zu stellen. Bei ihnen handelt es sich um die Generation der Nicht-Mehr-Eingestiegenen. Ihre Grundhaltung zur DDR war die des „Protestes durch Verweigerung " ${ }^{\text {i }}$. Ihre Lust am direkten Eingreifen in den taglichen Ablauf der Gesellschatt war sichtlich gebremst. Diese Generation trat den „Rückzug statt (der) Offensive" an, wic es der Dichter Kurt Drawert (geb. 1956) für sich und seine Altersgleichen treffend auf den Punkt brachte: „... im Osten und doch nicht im Osten, wir wohnten, aber wir lebten nicht dort ${ }^{482}$ - eine Aussage, die nachhaltig von Ergebnissen der Jugendforschung gestützt wird: Ab Ende der 70er Jahre ist eine Stagnation in der Bewußtseinsbildung Jugendlicher in bezug auf alle wichtigen

\footnotetext{
79 Stefan Wolle, Die "nachvollziehende Rebellion“ der DDR-Achtundsechziger, in: Leviathan 4 (Dezember 1998) 530.

80 Heinz Bude in einem Gespräch mit Rudolf Woderich über Biographieforschung im neuen Osten und im alten Westen, Dynamische Gelegenheitssucher und defensive Einfädler, in: Berliner Debatte INITIAL 2 (1966) 5.

${ }^{81}$ Günther Lange, Hans-Jörg Stiebler, Abschied von der Utopie? Jugend in der DDR im sozialen Wandel der achtziger Jahre, in: Sozialisation im Sozialismus, hrsg. v. Gunther Burkart (ZSE, Zeitschrift für Sozialisationsforschung und Erziehungssoziologie, 1. Beiheft [1990]) $55-69$.

82 Kurt Drawert, Rückzug statt Offensive, in: Freitag 52/1 (18. Dezember 1998).
} 
politischen Werte der Gesellschaft (Identifikation mit dem Marxismus-Leninismus und dem Staat DDR, Einstellung zur SED, zur Sowjetunion und zur historischen Perspektive des Sozialismus, Haltung zur Bundesrepublik) zu verzeichnen $^{83}$. Nur kurz unterbrochen durch die Nachrüstungsdebatte und den Hoffnungsschub auf eine Reformierung des Sozialismus, der sich mit Gorbatschows Politik von Glasnost und Perestroika auch für viele Jugendliche verband, spiegelt sich in den Daten soziologischer Untersuchungen ab 1987/88 pure Endzeitstimmung wider. „Die regressiven Tendenzen gingen geradezu in einen Verfall der Identifikation mit den sozialistischen Werten über ... " ${ }^{84}$ Potenziert wurde diese Entwicklung noch durch einen tiefgreifenden Wandel der jugendlichen Lebensorientierungen, der - so Walter Friedrich - „eher als "Mentalitätsumbruch" oder "Werteaufbruch" in der DDR charakterisiert werden kann" ${ }^{85}$. Das Streben der Jugendlichen nach Selbstgestaltung und sozialer Anerkennung nahm seit 1975 stark zu. Sprunghaft stieg das Erlebnisstreben, die Orientierung auf Mode, Luxus und Geselligkeit: alles Reaktionen auf Defizite des DDR-Alltags, aber auch Wunschprojektionen eines Gesellschaftsbildes, das sich ganz selbstverständlich der (medienvermittelten) Vorteile des Westen bediente. In dieser Generation tritt „die systemimmanente Denkweise ... zurück zugunsten einer moralischen und in gewissem Sinne auch ästhetischen Kritik" am SED-Staat ${ }^{86}$. Damit enthob sich diese Jugendgeneration lange selbst einer direkten politischen Wirksamkeit: „Sie forderten den Staat und seine Instanzen nicht heraus, sondern ignorierten ihn und traten den langen Marsch durch die Institutionen erst gar nicht an. ${ }^{\text {87 }}$ Auf ihrer Agenda standen statt dessen das Streben nach individuellem Wohlstand, kultureller (Szenen-)Vielfalt und geistigem Freiraum, wo auch immer die schwächelnde DDR ihn nicht mehr verhindern konnte.

Die dominierende Generationsgestalt der End-DDR nenne ich daher Distanzierte Generation. Mit ihrer Dominanz vollzog sich ab 1975 in der Abfolge der Jugendgenerationen der DDR erstmals ein (folgenschwerer) Paradigmawechsel. Nicht mehr die systemfreundlichen Strömungen stellten die prägende Generationseinheit unter den Jugendlichen, sondern jene, die ihr kritisch-distanziert bis ablehnend gegenüberstanden. So machte (auch und gerade) die Jugend der DDR den Weg in die demokratische Revolution vom Herbst 1989 frei. Zwar mag es vom Durchschnittsalter der führenden Köpfe der Bürgerbewegung her Sinn machen, von 1989 als einer "Revolution der Vierzigjährigen " 88 zu sprechen. Der Kern der Leipziger Montagsdemonstranten lag mit 25 bis 55 Jahren ebenfalls jenseits des Jugendalters ${ }^{89}$. Doch ändert sich das Bild schlagartig, wenn wir die

$83 \mathrm{Vgl}$. Walter Friedrich, Mentalitätswandlung der Jugend in der DDR, in: SAPuZ B 16-17 (13. April 1990) 25-37; im folgenden zitiert: Friedrich, Mentalitätswandlung.

84 Förster, Politisches Bewußtsein 79.

85 Friedrich, Mentalitätswandlung $34 \mathrm{f}$.

86 Geulen, Politische Sozialisation 329.

87 Wolle, Heile Welt 232.

88 Wierling, Opposition und Generation.

89 Lindner, Demokratische Revolution 114. 
Massenflucht von DDR-Bürgern über Ungarn und die bundesrepublikanischen Botschaften in Prag, Warschau und Sofia in den Westen als weiteren entscheidenden Auslöser für den Kollaps des SED-Staates einbeziehen. Das Durchschnittsalter der 225233 Flüchtlinge und Übersiedler, die vom 1. Januar bis 9 . November 1989 aus der DDR in Bundesrepublik kamen, lag bei ca. 23,5 Jahren ${ }^{90}$. Als sich diese erst nach dem Mauerbau geborenen „Kinder der geschlossenen Gesellschaft" 91 entschlossen, der DDR den Rücken zu kehren, war es kaum mehr ein Abschied von „ihrem Land“, sondern einer von einem jeweils konkreten sozialen Umfeld, von Familie und Freundeskreis. Die „Generation ohne Alternative ${ }^{\text {92 }}$ schuf sich eine solche - durch ihren Ausbruch aus dem DDR-System bzw. ihre Emanzipation davon.

Parallel zu ihr existierte im gleichen Zeitraum aber weiterhin auch eine Generationseinheit, die sich konform zum Staat verhielt. Die Loyalität zur DDR und deren Institutionen hielt sich bei ihnen allerdings in Grenzen - zur gegenseitigen Vorteilsnahme. Kehrten viele von ihnen z. B. "spätestens nach dem Erreichen ihres beruflichen Ziels der FDJ den Rücken "93, ließ sie der Jugendverband - wegen des schönen Scheins - dennoch weitgehend in Ruhe. Die Kraft, Einfluß auf das Gros der Jugend zu nehmen, hatte die FDJ längst verloren. Auch der Versuch, sich mit bis dato in der DDR nicht zugelassenen Open-Air-Konzerten westlicher Rockstars wie Bob Dylan, Joe Cocker oder Bruce Springsteen einen Prestigegewinn bei der Jugend $\mathrm{zu}$ „erkaufen “, fruchtete nur noch wenig”. Die Jugendlichen strömten zwar in Scharen zu den Konzerten, aber nicht mehr in die FDJ-Versammlungen.

\section{Erstes Fazit und offene Felder}

Das Projekt der politischen Vereinnahmung der Jugend für den Sozialismus war in der DDR lange vor dessen Ende gescheitert. Zu fragen bleibt, ob dies auch an den hier dargestellten Jugendgenerationen gelegen hat. Zwar konnten sie prägende Gestalt für ihre jeweilige Generation erreichen; politisch innovativ im Sinne des Systems wurde jedoch lediglich die Aufbaugeneration - soweit dies die „Ge-

90 Lindner, Demokratische Revolution 46. $90 \%$ der Flüchtlinge des Sommers 1989 waren unter 40 Jahre alt, was dafür spricht, daß sich in der Endzeit der DDR auch nennenswerte Teile der Integrierten Generation aus ihrer Bindung an diesen Staat zu lösen begann und/ oder vom Sog der Massenflucht erfaßt wurde.

91 Zwabr, Umbruch 443.

92 So nennt Bettina Völter in ihrem gleichnamigen Aufsatz die nach dem Mauerbau geborene Jugendgeneration, weil sie ${ }_{n}$ die deutsche Teilung ... bei ihrer Geburt vorfanden bzw. sie als alternativlose gesellschaftliche Realität erlebten." In: Berliner Debatte INITIAL 7 (1996) 6, 109.

93 Wolle, Heile Welt 114.

$94 \mathrm{Vgl}$. Michael Raubut, Musik kennt keine Grenzen. Politische Hintergründe internationaler Rockkonzerte in der DDR der Endachtziger, in: Helga Gotschlich (Hrsg.), ,Links und links und Schritt gehalten ...“. Die FDJ: Konzepte - Abläufe - Grenzen (Berlin 1994) 304-310. 
neration der Alten“ zuließ. Die Integrierte Generation dagegen konnte Innovationen im Sinne gesellschaftlicher Veränderungen weder für noch gegen das DDRSystem dauerhaft ausprägen. Sie paralysierte sich durch ihr Streben nach Übereinstimmung weitgehend selbst. Aber auch die Distanzierte Generation vermochte lange keine eigenständigen politischen Zeichen zu setzen. Erst als sie - zu nicht unerheblichen Teilen - ihre Existenz in diesem System auch physisch in Frage stellte, nahm sie unübersehbar Einfluß auf das Ende der DDR.

$\mathrm{Zu}$ lange war das politische Feld uneingeschränkt von jener Generation besetzt, die diesen Staat bereits gegründet hatte. Zweifelsohne ist Mühlberg zuzustimmen, dem zufolge „Generationen dann wirksame Verbände werden, wenn die kulturellen Konkurrenzen zwischen sozialen Bewegungen und Strömungen bedeutungsvoller, die Klassenkonkurrenzen dagegen schwächer werden "95. Das hier vorgestellte Modell der politischen und sozialen Jugendgenerationen der DDR bedarf also unbedingt der Ergänzung um eine kulturelle Perspektive. Dies ist andernorts bereits ansatzweise geschehen". An dieser Stelle kann - aus Platzgründen - lediglich darauf verwiesen werden, daß im kulturellen Feld die Parallelen zur Entwicklung der Altersgleichen in der Bundesrepublik deutlich größer sind als im politischen Raum. Die kulturelle Identität Jugendlicher in der DDR war immer schon eine Mischidentität, von Elementen des Ostens ebenso geprägt wie von denen des Westens, und zwar mit wechselnden Dominanzen: ein Unikat mit vielen Anleihen! Sie bei weiteren Analysen der Jugendgenerationen stärker zu betonen, ist daher auch eine Forderung der Gegenwart!

Bleibt die nicht unerhebliche Frage, wie jene Jugendgeneration zu charakterisieren ist, die zwar noch in der DDR geboren wurde, deren Eintritt in das Jugendalter jedoch erst mit oder nach dem Ende der DDR erfolgte. Es entspricht der Logik des dargestellten Generationsmodells, daß die nach 1975 Geborenen, deren erste Kohorte genau 1989/90, an der Bruchstelle der Systeme, in die Jugend bineinwuchs, die Wendezeit als einen deutlichen Einschnitt in ihre Sozialisation erlebten. Allerdings hatten sie gegenüber allen anderen beteiligten Generationen den Vorteil, sich unbelasteter dem Wendeprozeß stellen zu können. Zugleich befanden sie sich in einer Lebensphase, in der sie wie jeder Heranwachsende (zu allen Zeiten) verstärkt auf die Hilfestellung von Erziehungsautoritäten - Personen wie Institutionen - angewiesen waren. Doch: Ob Eltern oder Verwandte, Schulen, Jugendorganisationen oder Medien - sie alle fielen im Osten dafür weitgehend aus! Nicht nur, weil ihnen die Lebensumstände der Bundesrepublik ebenfalls fremd waren, sie also schon faktisch nicht in der Lage waren, den Heranwachsen-

95 Müblberg, 68 im Osten.

\% Vgl. Bemd Lindner, Sozialisation und politsche Kultur junger Ostdeutscher vor und nach der Wende - ein generationsspezifisches Analysemodell, in: Uta Schlegel, Peter Förster (Hrsg.), Ostdeutsche Jugendliche: Vom DDR- zum Bundesbürger (Opladen 1996) 31-34. Ein Ubberblick über wichtige Entwicklungslinien der DDR-Jugend in ausgewählten kulturellen und künstlerischen Feldern findet sich bei: Bernd Lindner, Dieter Wiedemann, Kultur und Medienforschung, in: Friedrich, Das Zentralinstitut 301-351. 


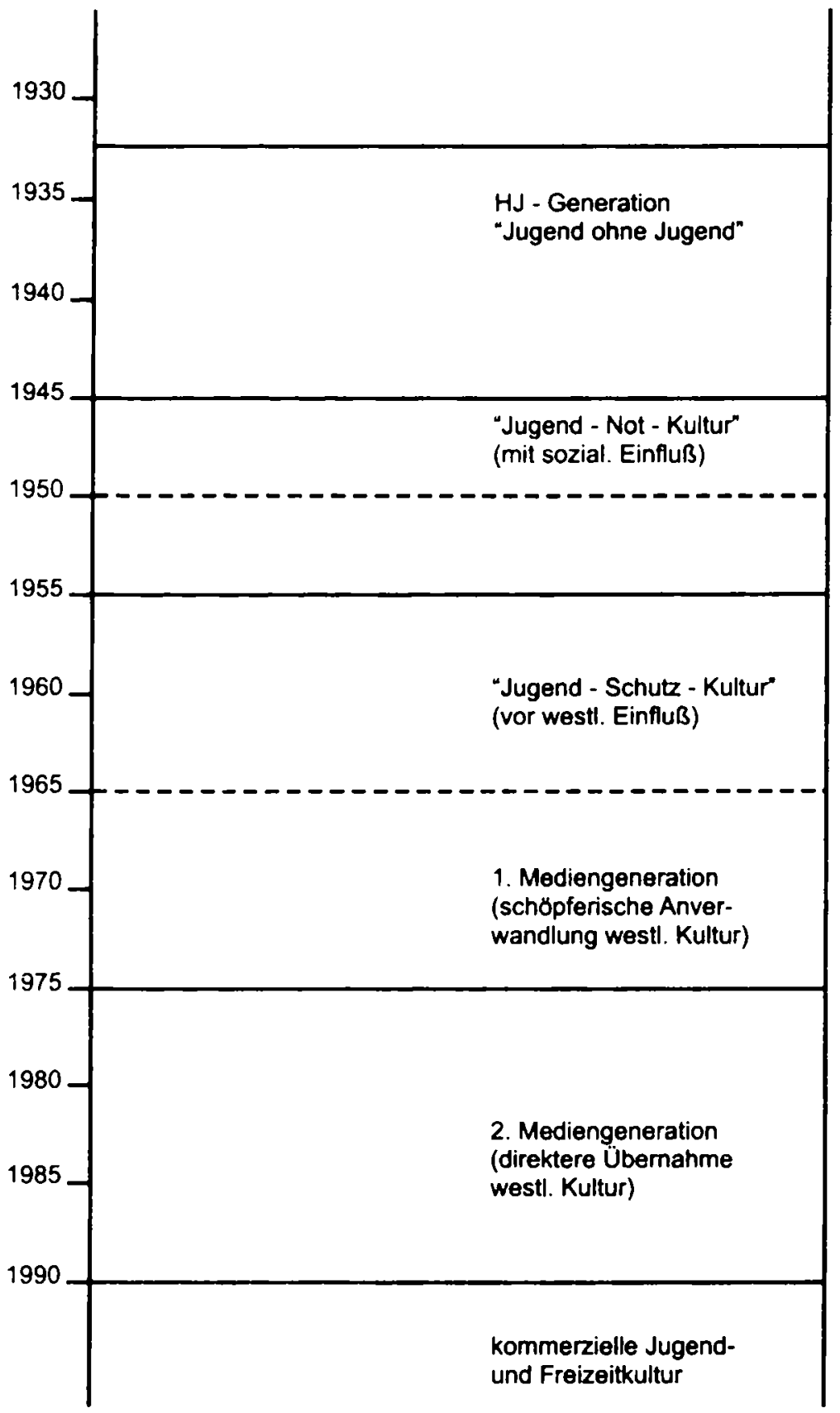

Abb. 3 
den Ratschläge zu erteilen, sondern vor allem auch, weil ihre Glaubwürdigkeit in den Augen der Jugendlichen in hohem Maße diskreditiert war. Das galt zwar nicht in gleichem Maße für die Kirche97, doch war und ist deren Wirkungsraum durch die hochgradige Säkularisierung des Ostens unter Jugendlichen erheblich eingeschränkt ${ }^{98}$. Aber auch die eingeführten Institutionen und Organisationen der Jugendarbeit des Westens konnten hier nur bedingt als Äquivalent wirksam werden: Zu unbekannt waren ihre Strukturen, zu fremd ihre Genese und nicht zuletzt zu ungeschickt ihre Versuche, im Osten Fuß zu fassen.

Dementsprechend waren (und sind) die Heranwachsenden im Osten weitgehend auf sich selbst verwiesen. Wir haben es bei ihnen mit einer in hohem Maße Unberatenen Generation zu tun ${ }^{99}$. Die Folgen dieses Sachverhaltes für diese Jugendgeneration, die heute noch anhalten, sind bereits allenthalben zu spüren: Distanzierung und soziale Entwurzelung, Zuwachs an Aggressivität und Gewalt sowie politisches Desinteresse auf der einen und politische Polarisierung an rechten und linken Rändern auf der anderen Seite. Erschwerend kommt hinzu, daß es sich bei ihnen weitgehend um die Kinder der Integrierten Generation handelt, jener Jugendgeneration der DDR also, die es nach der "Wende“ mit Abstand am schwersten hatte, in der bundesrepublikanischen Gegenwart anzukommen. Mit den "guten Zeiten" der DDR-Entwicklung besonders eng verbunden, kompensiert diese Generation ihre sozialen und mentalen Probleme mit der Gegenwart verstärkt durch ( $n$ )ostalgische Rückbezüge, die um so stärker auch auf ihre Kinder

97 Sie hatte durch ihre Schlüsselfunktion im Herbst 1989 sogar einen erheblichen Imagegewinn in der Bevölkerung zu verzeichnen, den sie aber nicht dauerhaft für sich nutzen konnte. Vgl. Matthias Hartmann, Kirchen, in: Wermer Weidenfeld, Karl-Rudolf Korte (Hrsg.), Handbuch zur deutschen Einheit (Bonn 1996) 419-428.

$98 \mathrm{Vgl}$. Walter Friedrich, Weltanschauliche Positionen der Jugend, in: ders., Das Zentralinstitut 184-205.

99 Ihre Charakterisierung in den Medien als "Verwahrloste Generation" ("die ihre Prägung in einer gesellschaftlichen $\mathrm{Z}$ wischenzeit erfahren ${ }^{*}$ hat, als ,alle bekannten Werte der DDR weggebrochen" und die neuen "noch fremd" waren, in: Freitag 19 [1998]), als "Generation Null“ ( $z u$ alt, um ihre Vergangenheit zu vergessen, und zu jung, um in ihr zu verharren “, in: Tagesspiegel v. 22.10.1998) oder Generation nauf dem Seil “ (die zwischen Mitleid mit ihren Eltern und den eigenen, brüchigen Zukunftshoffnungen schwankt, in: Die Zeit 38 [1999]) spricht ebenso dafür wie umfangreiche soziologische Befunde aus dem ersten Jahrzehnt nach der Wiedervereinigung. Vgl. dazu u. a.: Imbke Bebnken, Bernd Lindner, Jürgen Zinnecker u. a., Schüler-Studie '90. Jugendliche im Prozeß der Vereinigung (Weinheim, München 1990); Peter Förster, Walter Friedrich, Wilfried Schubarth, Jugend Ost. Zwischen Hoffnung und Gewalt (Opladen 1993); Walter Bien, Ute Karig u. a., Cool bleiben - erwachsen werden im Osten (Weinheim, München 1994); Hans-Jürgen von Wensierski, Mit uns zieht die alte Zeit. Biographie und Lebenswelt junger DDR-Bürger im Umbruch (Opladen 1993); Rainer Silbereisen, Laszlo $A$. Vaskovics, Jürgen Zinnecker (Hrsg.), Jungsein in Deutschland. Jugendliche und junge Erwachsene 1991 und 1996 (Opladen 1993); Walter Friedrich, Peter Förster, Jugend im Osten. Politische Mentalität im Wandel (Leipzig 1996); Schlegel, Förster, Vom DDR- zum Bundesbürger, insbesondere 21-388; Peter Förster, Die 25jährigen auf dem langen Weg in das vereinte Deutschland, in: APuZ B 43-44 (1999) 22. Oktober, 20-31 sowie die Shell-Jugendstudien 1992, 1997 und 2000 (alle Opladen). 
und Enkel (ein)wirken, als diese selbst Probleme mit ihrer Verortung in der Bundesrepublik des 21. Jahrhunderts haben"7. Die Geschichte der Jugendgenerationen der DDR setzt sich also bis in die unmittelbare Gegenwart fort, obwohl der Staat, der sie hervor- und den sie mit zu Fall gebracht haben, schon über ein Jahrzehnt nicht mehr existiert. Die Ursachen dafür und Reaktionsmuster darauf zu erhellen, ist eine Möglichkeit des hier vorgestellten Generationenmodells und zugleich eine Leistung, die es noch zu erbringen hat.

97 Vgl. Bernd Lindner, Die Generation der Unberatenen, in: Berliner Debatte Initial 14 (2003) 2, 32 f. 
